\title{
Bewuchsuntersuchungen auf Natursteinsubstraten im Gezeitenbereich des Nordsylter Wattenmeeres: Sessile und hemisessile Tiere *
}

\author{
G. LuTHER \\ Biologische Anstalt Helgoland (Litoralstation); \\ List/Sylt, Bundesrepublik Deutschland
}

\begin{abstract}
Fouling studies on natural-stone substrates in the tidal zone of the North Sylt wadden sea: Sessile and hemisessile animals. Six different types of test substrates, arranged in order of texture from smooth (Solnhofen limestone, Bunter sandstone, and basalt) to rough surfaces (Middle Triassic limestone, granite, and basaltic lava), were exposed in the tidal zone of the wadden sea near the harbour of List (Island of Sylt, North Sea). The test substrates were fixed to panels at the midtide to high-water level, the midtide to low-water level, and $75 \mathrm{~cm}$ below the latter (sublittoral level). Animal settlement was primarily influenced by abiotic factors at the two higher levels. In the sublittoral zone, however, influences of biotic factors (competitors and predators) predominated. Thereforc, the physical quality of the chosen substrates more decisively affected the growth of settling animals above the midtide to lowwater mark than in the deeper zone. At the midride to high-water level Buldanus buthanoides only settled on the rough surface of the Middle Triassic limestone and in the troughs of the Bunter sandstone; barnacles attached to the even surface of the latter, were destroyed by wave beating. They died on the rough surface of the dark-coloured granite, when this stone was warmed during low-water. At the midtide to low-water level, the barnacles survived best on the rough surface of the Middle Triassic limestone and the granite. Only the larvae of $B$. crenatus and $B$. improvisus preferred to settle on the even surface of the Bunter sandstone and the basalt; but there they died from wave beating or desiccation. The surface of the basaltic lava, formed by sharp-edged pores, was unsuitable for settling of barnacles. Colonies of Hydrozoa covered each substrate with a somewhat rough surfacc structure, cven the basaltic lava. At the sublittoral level, differences in settling between the single substrates disappeared more and more. Nevertheless, the three species of barnacles showed the same preferences in settling, as they did at the higher levels. In August, Asterias rubens destroyed all barnacles and thus restricted the lower limit of barnacle settlement at the low-water mark. The distribution of epibiotic organisms is dependent upon the density of their "living substrates", directly attached to the stone surfaces. During the short time of their growth, hemisessile young Mytilus edulis settled on thready forms like algae, or on raised areas like the top of barnacle shells. Therefore, young mussels could be found on stones, that already carried a compact cover of Enteromorpha sp. or a dense settlement of adult barnacles. The polychaetc Polydora ciliata rarely burrowed directly into stony substrates (Middle Triassic limestone, Solnhofen limestone). It settled primarily between barnacle shells where it was sheltered from wave beating and at the same time profited from the current produced by the filter-fecding organs of the barnacles. The density of this polychaete was directly proportional to the density of the barnacles. After
\end{abstract}

* Herrn Professor W. E. Ankel zur Vollendung des 80. Lebensjahres in Dankbarkeit gewidmet. 
A. rubens had destroyed the barnacles in the sublittoral zone, $P$. ciliata disappeared too. At the low-water mark, however, the $P$. ciliata population between living and active barnacles increased. Hence, the lower limit of $P$. ciliata - as those of barnacles and mussels - was fixed by this predator.

\section{EINLEITUNG}

Die Struktur des Lebensraums übt einen entscheidenden Einfluß auf die Zusammensetzung der in ihm lebenden Organismen aus. Bei benthischen Formen ist die Art des Wohnsubstrats für die Besiedlung bestimmend. Auf verschiedenen Hartsubstraten können sich sehr unterschiedliche Tiergemeinschaften entwickeln. Die vorliegende Untersuchung liefert ein Beispiel für diese Zusammenhänge. Um derartige Biozönosen $\mathrm{zu}$ untersuchen, wurden sechs natürlich gewachsene Gesteine verschiedener Herkunft in drei Positionen am Anleger der Autofähre unterhalb des Hafens von List auf Sylt (östliche Nordsee) angebracht. Die ausgewählten Substrate - Solnhofener Plattenkalk, Muschelkalk, Buntsandstein, Granit, Säulenbasalt und Basaltlava - variierten je nach Farbe, Härtegrad, Oberflächenbeschaffenheit, Korngröße und Porosität. In einer früher erschienenen Veröffentlichung wurde berichtet (Luther, 1976b), wie weit die genannten physikalischen Eigenschaften den Algenbewuchs beeinflussen. Bei diesen Studien zeichneten sich auch Unterschiede in der Abundanz tierischer Organismen $a b$, die sich auf die Struktur der verwendeten Substrate zurückführen ließen. Die Lage des Untersuchungsortes, die Position der Versuchsreihen, die Wirkung abiotischer und biotischer Faktoren sowie methodische Einzelheiten sind in früheren Arbeiten (Luther, 1976a, b) eingehend beschrieben worden.

\section{VERTIKALE VERTEILUNG DER MAKROFAUNA}

Die Zonierung der Makrofauna im Bereich des Tidenhubs $(1,70 \mathrm{~m})$ ist in $\mathrm{Ab}$ bildung 1 dargestellt. Der Cirripedier Balanus balanoides dominierte im Raum oberhalb, $B$. crenatus und $B$. improvisus unterhalb der Mitteltiden-Niedrigwasserlinie. Auf dem Hochwasserniveau war B. balanoides der einzige Vertreter sessiler tierischer Organismen. Im Bereich der Mitteltiden-Niedrigwasserlinie konnten sich außerdem weniger robuste Formen entwickeln wie Polydora ciliata und Lanice concbilega, Mytilus edulis, Coryne sp. und Tubularia larynx, Laomedea longissima und L. flexuosa. Die gleichen Arten waren im Bereich ständiger Wasserbedeckung zu finden mit einer im Vergleich zur Mitteltiden-Niedrigwasserlinie unterschiedlichen Besiedlungsdichte. Daneben traten Metridium senile und Aiterias rubens auf. Obwohl diese Seesternart zu den vagilen Formen gehört, mußte sie berücksichtigt werden, da sie durch ihre räuberische Lebensweise das Siedlungsbild verändern konnte.

Bei der Besiedlung der Versuchssubstrate durch tierische Organismen zeigten sich zwei auffallende Unterschiede zum Bewuchs der unmittelbaren Umgebung: Mytilus edulis war nur während eines hemisessilen Jugendstadiums in großer Anzahl vorhanden, ohne (bis auf wenige Ausnahmen) danach auf den Versuchssubstraten zur sessilen Lebensweise überzugehen. Littorina littorea, die massenhaft im Untersuchungs- 


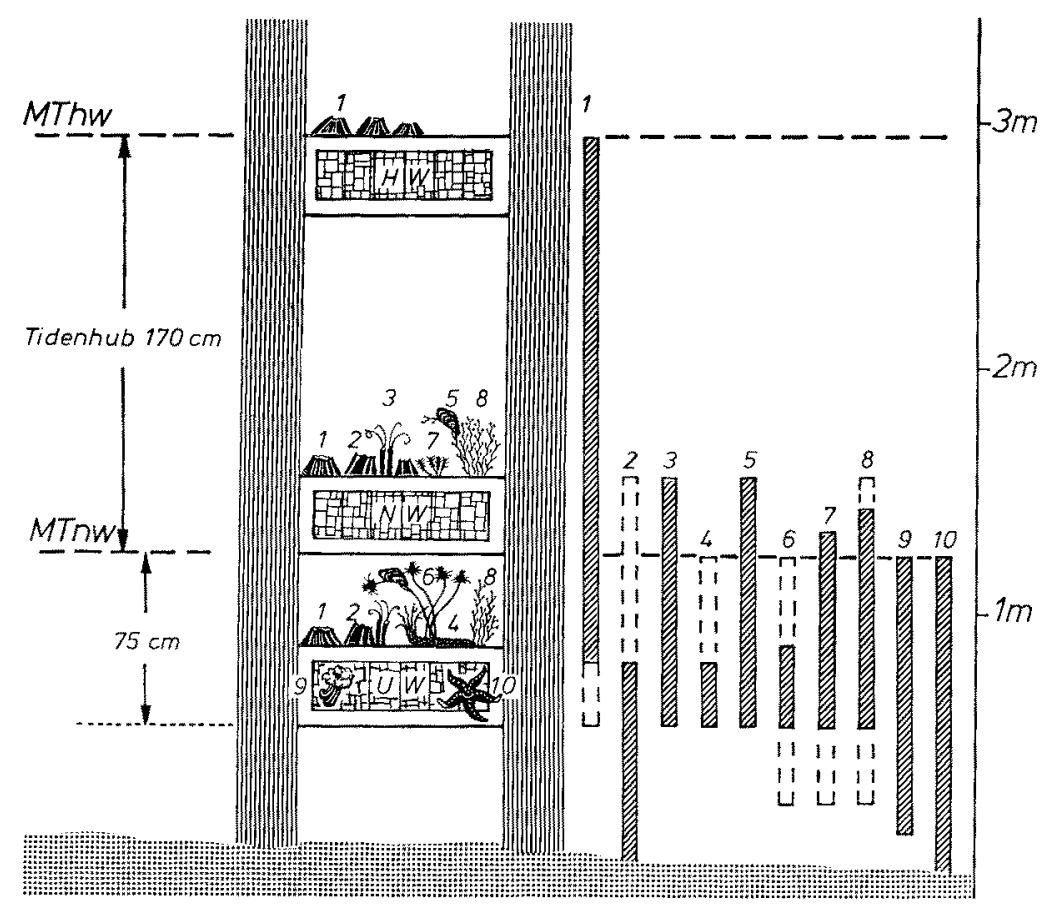

Abb. 1: Versuchsgestell und Versuchsreihen auf dem Hochwasserniveau (HW), Niedrigwasserniveau (NW) und Unterwasserniveau (UW). Die generelle Zonierung der auf den Versuchssubstraten angesiedelten sessilen und hemisessilen Tiere ist durch Vertikalkolumnen angedeutet (schraffert: häufig; gestrichelt: selten). 1 Balanus balanoides, $2 \mathrm{~B}$. crenatus und $\mathrm{B}$. improvisus, 3 Polydora ciliata, 4 Lanice conchilega, 5 Mytilus edulis, 6 Tubularia larynx, 7 Coryne sp., 8 Laomedea sp., 9 Metridium senile, 10 Asterias rubens

gebiet vorkommt, ließ sich selten auf den Versuchssubstraten beobachten. Vermutlich erschwerte die Art, in der die Versuchsreihen angebracht waren, der Schnecke den Weg zu den Substraten.

\section{METHODIK}

Die Versuchsreihen auf den ausgewählten Niveaus unterschieden sich in je eine Kurzzeit- und eine Langzeitserie. Die Kurzzeitserien boten, da die bodenreifen Larven durch das häufige Auswechseln der Substrate genügend Raum zum Ansetzen vorfanden, einen Uberblick über die möglichen Besiedlungsanteile. Auf der Langzeitserie war die Sukzession im Ablauf eines Jahres zu verfolgen. Die Auszählergebnisse wurden mit Hilfe eines Kartiernetzes im Größenverhältnis 1:1 auf Millimeterpapier ibertragen (Abb. 2). Balaniden, Polychaeten und Muscheln konnten nach Stüickzahl $\left(\mathrm{n} \mathrm{cm}^{-2}\right)$ erfaßt werden. Bei den sich rasenartig ausbreitenden Kolonien mancher Hydrozoen erschien das Auszählen nach dem Grad der Besiedlungsdichte pro $\mathrm{cm}^{2}$ (Methode nach Braun-Blanquet) vorteilhafter. 


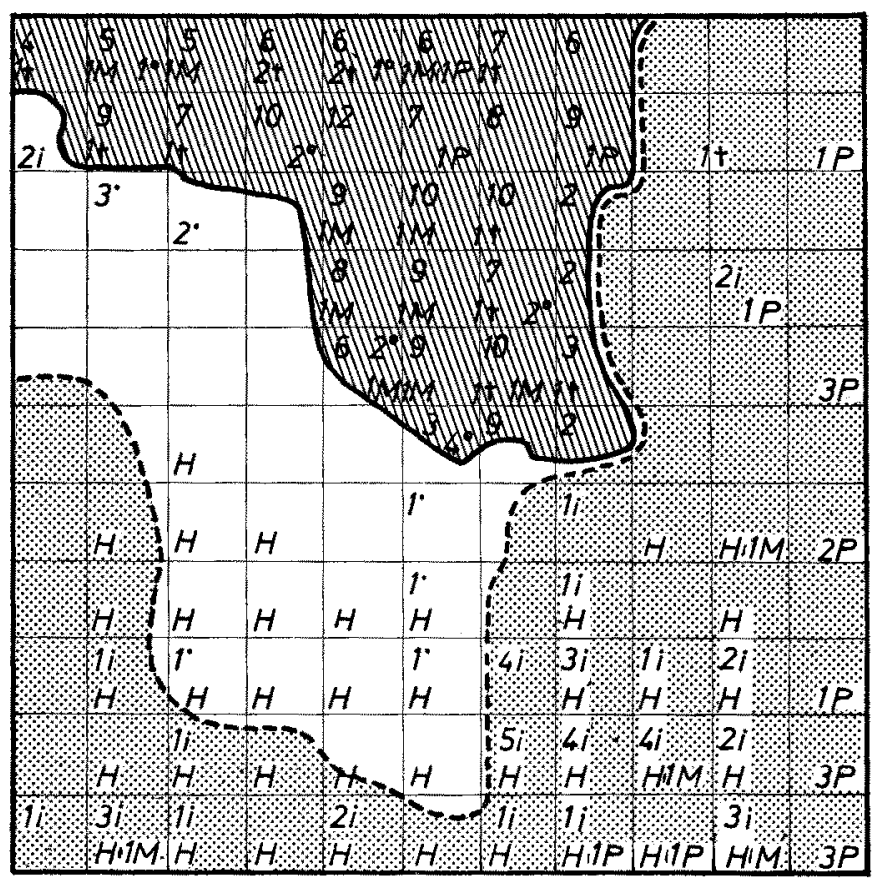

Abb. 2: Protokoll des Versuchsfeldes von Solnhofener Plattenkalk, Langzeitserie, eingesetzt am 24. März 1972, Kontrolle vom 31. Juli 1972. Schraffiert: $29 \mathrm{~cm}^{2}$ unbeschädigter Substratoberfläche mit der Frühjahrsbesiedlung von 206 Balanus balanoides (Zahl ohne Symbol) und 12 abgestorbenen Individuen (Zahl mit Symbol Kreuz), 12 Polydora ciliata (Zahl mit Symbol Kreis), 10 Mytilus edulis (Zahl mit Symbol M) und 3 Porphyra purpurea (Zahl mit Symbol P). Punktiert: $61 \mathrm{~cm}^{2}$ seit Ende Juni erodierter Substratoberfläche mit der Neubesiedlung von Enteromorpha sp., 45 Balanus improvisus (Zahl mit Symbol i), 4 Mytilus edulis, 16 Porphyra purpurea und $27 \mathrm{~cm}^{2}$ mit maximaler Besiedlungsdichte von Laomedea sp. (Symbol H). Weiß: $32 \mathrm{~cm}^{2}$ seit Mitte Juli erodierter Substratoberfläche mit der Neubesiedlung von 9 jungen $B$. improvisus (Zahl mit Symbol Punkt) und $12 \mathrm{~cm}^{2}$ mit maximaler Besiedlungsdichte von Laomedea sp.

Neben den Serien, die die 6 ausgewählten Natursteinsubstrate enthielten, wurden in gleicher Höhe solche mit künstlich hergestellten Substraten aus Quarzsand angebracht. Der Durchmesser der Sandkörner umfaßte 5 Fraktionsbereiche: < 0,25; $0,25-0,5 ; 0,5-1,0 ; 1,0-2,0 ; 2,0-4,0 \mathrm{~mm}$ (nähere Angaben vgl. Luther, 1976b).

\section{ERGEBNISSE}

Tierische Besiedlung auf dem Hochwasserniveau

Auf dem Hochwasserniveau hängt die Eignung von Natursteinen für eine tierische Besiedlung weitgehend von physikalischen Faktoren der Substrate ab wie Größe und Struktur des Porenraums und der damit verbundenen Kühlung der Oberfläche 
durch Wasserverdunstung, Oberflächenstruktur und Farbe des Gesteins. Die abiotischen Verhältnisse des Gezeitenbereichs bestimmten während der Versuchsjahre direkt oder indirekt über die Eigenschaften der Gesteine die Zusammensetzung und Abundanz der Makrofauna. Zum Beispiel fehlten die Balaniden 1971 auf dem Hochwasserniveau vollkommen. 1972 waren sie auf einigen Versuchssubstraten bis an die obere Grenze zu finden (Tab. 1).

Tabelle 1

Balanus balanoides. Besiedlungsdynamik anf dem Hodwasserniveau im Jahre 1972 (n pro Versuchsfläche). Zahlen in Klammern: die zusätzlich bei den Kontrollen jeweils neu vorgefundenen abgestorbenen Individuen

\begin{tabular}{|lccccc|}
\hline Substrat & 6. Juni & 18. Juli & 16. August & 31. Oltober 27. November \\
Solnhofener & 0 & & & & \\
Plattenkalk & 0 & 0 & 0 & 0 & 0 \\
Muschelkalk & 383 & 253 & 72 & 62 & 57 \\
Buntsandstein & $(38)$ & $(114)$ & $(181)$ & $(10)$ & $(10)$ \\
Granit & 56 & 59 & 44 & 35 & 34 \\
Säulenbasalt & $(1)$ & $(1)$ & $(15)$ & $(9)$ & $(1)$ \\
Basaltlava & 20 & 10 & 0 & 0 & 0 \\
\hline
\end{tabular}

Im Bereich der Mitteltiden-Hochwasserlinie, wo die Organismen von Wasserturbulenz und Austrodnung bedroht sind, bevorzugte Balanits balanoide: Substrate mit unregclmäßiger Oberfläche wic den zerklüfteten Muschelkalk, während er auf dem meist ebenen Buntsandstein ausschließlich in einer schützenden Vertiefung überlebte (Abb. 3). Bei Granit wurde die siedlungsbegünstigende Wirkung der unregelmäßigen Oberflächenstruktur durch die starke Erwärmung des Gesteins bei Sonnenbestrahlung aufgehoben. Hier starben alle angesetzten Exemplare bei einer ersten Hitzeperiode im Juli ab. Den gleichen Befund zeigten die Quarzsandsubstrate, auf denen sich Balaniden nur im Korngrößenbereich 2,0-4,0 mm angesiedelt hatten. Im allgemeinen fiel der Höhepunkt der Sterblichkeit mit dem Maximum der sommerlichen Luft- und Wassertemperaturen zusammen. Auf den ebenflächigen Gesteinen Solnhofener Plattenkalk und Säulenbasalt sowie auf der scharfkantigen, zerrissenen Oberfläche der Basaltlava konnten sich Balaniden im Bereich der Mitteltiden-Hochwasserlinie nicht entwickeln.

Im Jahr 1971 schienen die abiotischen Gegebenheiten eine Balanidenbesiedlung auf dem Hochwasserniveau verhindert zu haben. Die Versuchsreihen blieben dort in der Zeit, in der die Mehrzahl der bodenreifen Cypris-Larven ein Substrat aufsuchen (Ende April bis Anfang Mai), tagelang luftexponiert und waren starker Sonnenbestrahlung ausgesetzt (Abb. 4). Kräftige nordöstliche Winde mit durchschnittlich nur 50\% Luffeuchtigkeitsgehalt erhöhten die Gefahr des Austrocknens. Demgegenüber betrug die Luffeuchtigkeit im folgenden Jahr bei schwächeren westlichen Winden 


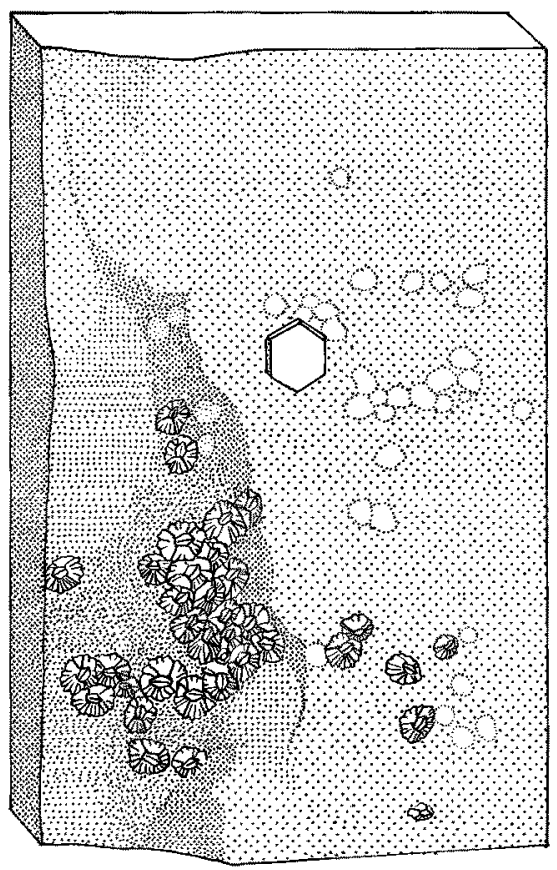

Abb. 3: Buntsandstein als Substrat auf dem Hochwasserniveau, eingesetzt am 24. März 1972, Zustand am 28. August 1972. Auf der planen, vertikalen Oberfläche - besonders dicht in der Nähe der sechseckigen Befestigungsschraube - sind fast nur Spuren der membranösen Basalplatten von Balanus balanoides sichtbar. Uberlebende Exemplare befinden sich vorwiegend in einer Vertiefung

$88 \%$. Die geringe Abundanz der Cypris-Larven im Plankton (vgl. Luther, 1976a) wirkte sich 1971 vermutlich gleichfalls ungünstig auf eine Besiedlung aus.

\section{Tierische Besiedlung auf dem Niedrigwasserniveau}

Im Bereich der Mitteltiden-Niedrigwasserlinie erhöhten sich Arten- und Individuenzahl der Makrofauna beträchtlich. Die Arten Balanus balanoides und B. crenatus traten bereits im April auf. Ihnen folgte ab Ende Mai $B$. improvisus und wenig später im Juni Mytilus edulis und Polydora ciliata. Lanice conchilega sowie thecate und athecate Hydrozoen waren erst im Juli zahlreicher zu finden. Die Befunde werden in gleicher Reihenfolge besprochen. Auf den Kurzzeitserien ließen sich die Arten gut beobachten, deren bodenreife Larven kurzfristig in großer Zahl auftreten (Balaniden, Miesmuscheln). Dagegen war die Besiedlung der Formen, die mehrere Generationen am Ort der ersten Anheftung entwickeln (Polydora ciliata) oder sich durch Knospung weiter verbreiten können (Hydrozoen), besser auf den Langzeitserien zu verfolgen.

$\mathrm{Kurzz}$ eits e rie n. Im allgemeinen bevorzugte Balanus balanoides auf dem Niedrigwasserniveau - ähnlich wie im Bereich der Mitteltiden-Hochwasserlinie - zum 

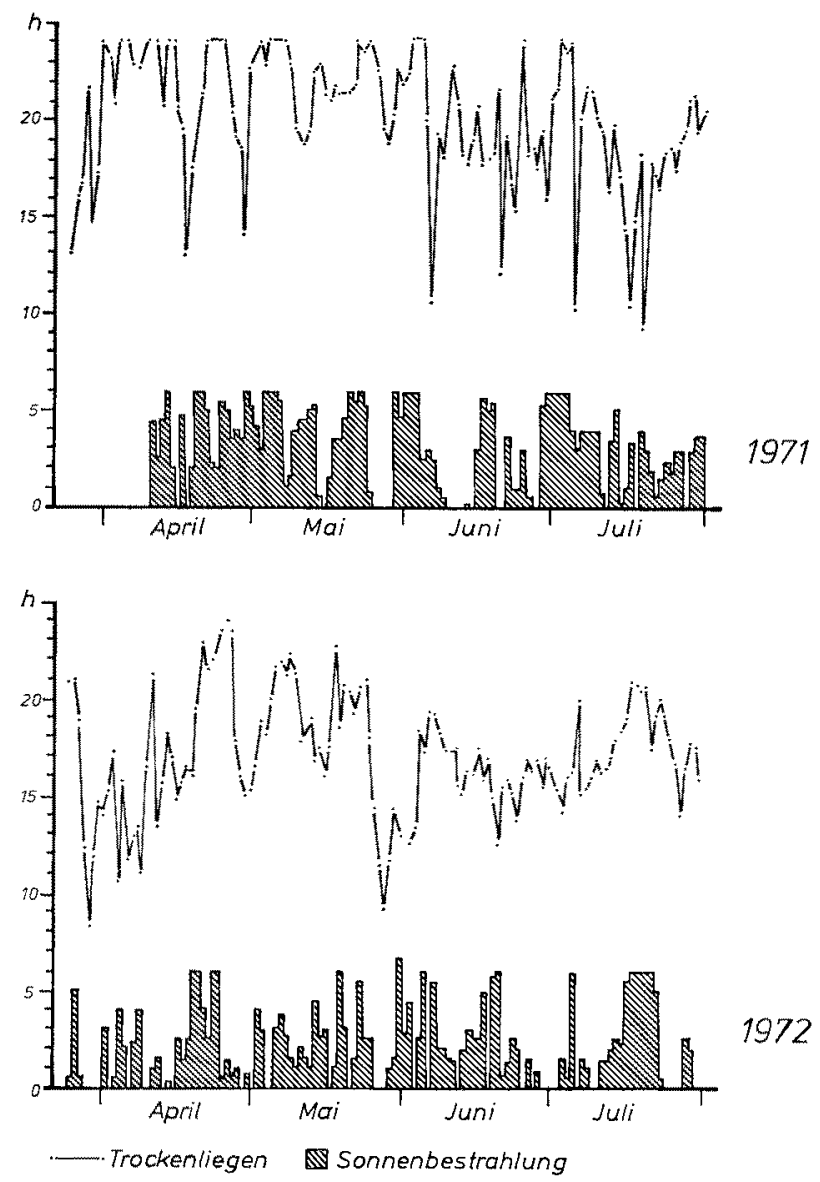

Abb. 4: Tägliches Trockenliegen der Substrate und direkte Sonnenbestrahlung während des Trockenliegens auf dem Hochwasserniveau von März bis Juli in den Jahren 1971 und 1972

Ansetzen Substrate mit unregelmäßiger Oberfläche, an erster Stelle Muschelkalk und Granit (Tab. 2). Substrate mit gleichmäßigen Oberflächen wie Buntsandstein, Säulenbasalt und Solnhofener Plattenkalk wurden dünner besiedelt. Basaltlava schien sich durch zahlreiche, scharfkantige Porenränder ungünstig auf das Anhetten der CyprisLarven auszuwirken. Als dagegen im Jahr 1972 verwittertes Material eingesetzt wurde, bei dem tonige Einschwemmungen und Ausfällungen eingedrungener Lösungen die Oberfläche verändert und die Porenränder entschärft hatten, entstand ein Effekt, der mehr dem der Muschelkalk-Oberfläche entsprach. Auf dem veränderten Material fand eine dichte Besiedlung statt, während sich auf dem unverwitterten, das 1971 und nach dem 4. Juni 1972 sowie 1973 benutzt wurde, nur wenige Balaniden ansetzten.

Die Bevorzugung des Muschelkalks als Besiedlungssubstrat ließ sich offensichtlich auf dessen unregelmäßiger, schrattiger Oberfläche zurückführen. Eine solche Ober- 


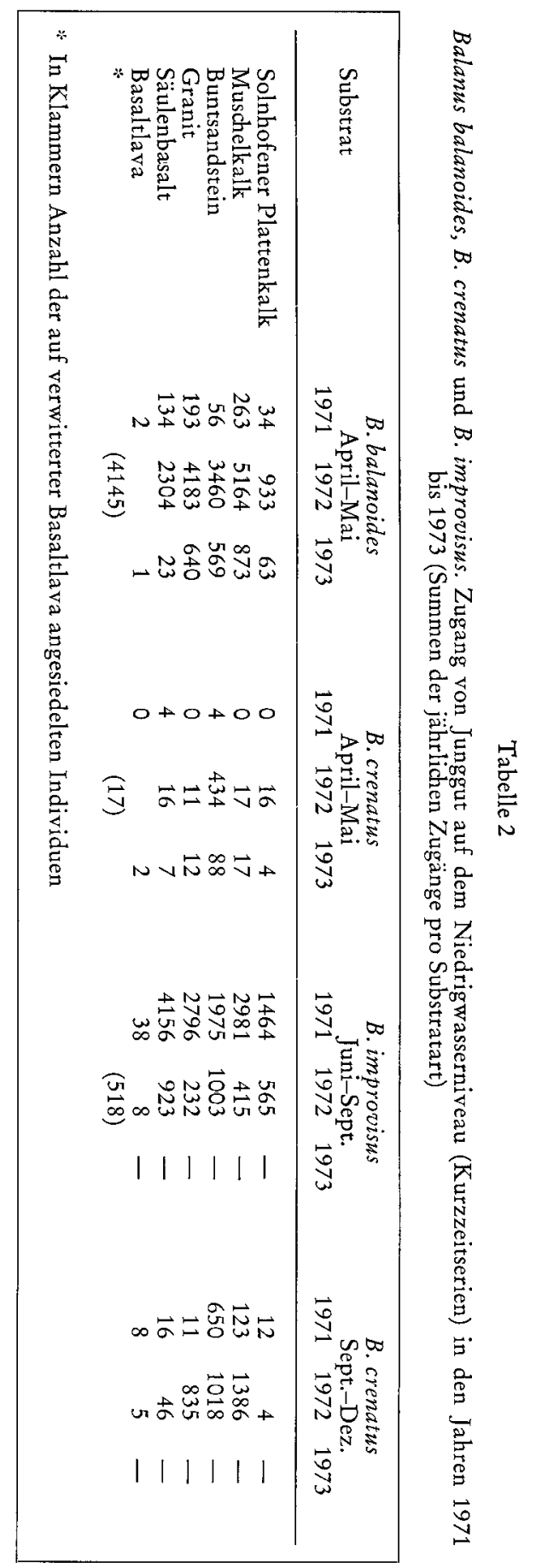


fläche kann besser als die glatter Gesteine ablaufendes Wasser beim Trockenfallen zurückhalten, und die gegen Austrocknung sehr empfindlichen Cypris-Larven und dünnschaligen Jungtiere der Balaniden vermochten daher leichter zu überleben.

Im Bereich der Mitteltiden-Niedrigwasserlinie zeigten sich bei den drei Balanidenarten in der "Wahl" der Substrate nur geringe Unterschiede. Zum Beispiel besiedelten Balanus crenatus und $B$. improvisus Gesteine mit glatten, regelmäßigen Oberflächen häufig stärker als $B$. balanoides. Dieses Siedlungsmuster traf jedoch nicht zu, wenn die Grünalge Enteromorpha sp. als Raumkonkurrent feinkörnige, regelmäßige Gesteine mit dichten Rasen überzog (Juni/Juli) und dort das Anheften von B. improvisus behinderte (Tab. 3). Mit der Dichte des Enteromorpba-Rasens verringerte sich die Zahl des letzteren auf den Substraten. Nur auf dem Buntsandstein entstanden in einigen schattigen Vertiefungen, die algenfrei geblieben waren, größere Siedlungsgruppen.

Tabelle 3

Enteromorpha sp., Mytilus edulis und Balanus improvisus. Abundanz (n pro Versuchsfäche) am 2. Juli 1971 (Kurzzeirserie auf dem Niedrigwasserniveau)

\begin{tabular}{|c|c|c|c|c|}
\hline \multirow[b]{2}{*}{ Versuchssubstrat } & \multicolumn{2}{|c|}{ Enteromorphasp. } & \multirow{2}{*}{$\begin{array}{c}\text { Mytilus } \\
\text { edulis } \\
\text { (Schalen- } \\
\text { länge } \\
1,5 \mathrm{~mm} \text { ) } \\
\mathrm{n}\end{array}$} & \multirow{2}{*}{$\begin{array}{c}\text { Balants } \\
\text { improvisus } \\
\text { (3-4 mm } \\
\text { basaler } \\
\text { Durch- } \\
\text { messer) } \\
\mathrm{n}\end{array}$} \\
\hline & $\begin{array}{l}\text { Bedeckungsanteil der } \\
\text { Versuchsfläche in cm" bei } \\
\text { ciner Bewuchsdichte von } \\
175 \text { Exemplaren } \mathrm{cm}^{-2}\end{array}$ & $\begin{array}{l}\text { Fadenlänge } \\
\text { nach } \\
\text { 18 Tagen } \\
(\mathrm{cm})\end{array}$ & & \\
\hline Solnhofener Plattenkalk & 90 & 2 & 1600 & 302 \\
\hline Muschelkalk & 120 & 2 & 3000 & 237 \\
\hline Buntsandstein & 120 & 7 & 4000 & 535 \\
\hline Granit & 30 & 1 & 102 & 1471 \\
\hline Säulenbasalt & 30 & 1 & 103 & 1672 \\
\hline Basaltlava & 120 & 5 & 3500 & 9 \\
\hline
\end{tabular}

Mytilus edulis erschien stets bei einer Größe von $0,5 \mathrm{~mm}$ und bevölkerte als frei beweglicher Benthosbewohner die Grünalgenrasen der Substrate. Dieses hemisessile Stadium der Jungmuscheln fiel zeitlich mit dem Maximum der Enteromorpha-Entwicklung zusammen, und mit Dichte und Länge der Algenfäden nahm die Besiedlung von Mytilus edulis zu, die von Balanus improvists dagegen ab (Tab. 3). Da Enteromorpha sp. vorwiegend auf fein-bis mittelkörnigen Substraten (Korndurchmesser $<1,0 \mathrm{~mm}$ ) gedieh (Luther, 1976b), waren dort die Jungmuscheln in großer Zahl anzutreffen. Raumkonkurrenz zwischen $B$. improvisus und Enteromorpba sp., dazu die Abhängigkeit der Jungmuscheln von fädigen Strukturen (Verwey, 1951; Bayne, 1964) bestimmten daher das Besiedlungsmuster. Uber eine Substratabhängigkeit bei der Besiedlung von Polydora ciliata und Hydrozoen ließ sich dagegen nichts aussagen, da die Zeitspanne, in der die Kurzzeitserien eingesetzt waren, für eine Entwidklung dieser Organismen nicht ausreichte.

L a ngzeitse rien. Die Wirkung von Brandung und von Tidenströmen ließ sich an den Substraten der Langzeitserien gut beobachten. Uber die Widerstands- 
fähigkeit der Gesteine gegen Abrasion wurde früher berichtet (Luther, 1976b). Die 1971 für die Untersuchung der Langzeitserie benutzte photographische Methode hatte sich als unzulänglich erwiesen. Sie ließ nur Schlüsse allgemeiner Art zu. Beispielsweise waren Balanidensiedlungen im Bereich der Mitteltiden-Niedrigwasserlinie auf Gesteinen mit unregelmäßiger Oberfläche (Muschelkalk, Granit) am beständigsten; Basaltlava zeigte sich dagegen trotz ihrer unregelmäßigen Oberfläche als besiedlungsfeindliches Material, das erst ab Oktober kleine Balanidengruppen aufwies. Da die prozentuale Verteilung der Arten nach der 1971 angewandten photographischen Methode nicht erfaßt werden konnte, enthält Tabelle 4 nur die Werte der Versuchsjahre 1972 und 1973.

Tabelle 4

Balanus balanoides, $B$. crenatus und $B$. improvisus. Besiedlungsdynamik auf dem Niedrigwasser-Niveau (Langzeitserien) in den Jahren 1972 und 1973 (Individuenzahl pro Versuchssubstrat)

\begin{tabular}{|c|c|c|c|c|c|c|c|c|}
\hline Substrat & \multicolumn{2}{|c|}{ B. balanoides } & \multicolumn{2}{|c|}{ B. crenatus } & $\begin{array}{c}\text { B. improvisus } \\
\text { Juni-Sept. } \\
1972\end{array}$ & $\begin{array}{c}\text { B. crenatus } \\
\text { Sept.-Dez. } \\
1972\end{array}$ & \multicolumn{2}{|c|}{$\begin{array}{c}\text { Uberlebende } \\
\text { am 21. Nov. } 1972\end{array}$} \\
\hline Solnhofener & & & & & & & & \\
\hline Plattenkalk & 1722 & 316 & 57 & 16 & 921 & 359 & 117 & 39 \\
\hline Muschelkalk & 2256 & 1675 & 115 & 22 & 346 & 193 & 609 & 2 \\
\hline Buntsandstein & 1549 & 1179 & 422 & 55 & 454 & 256 & 540 & $6 \overline{7}$ \\
\hline Granit & 1703 & 1111 & 82 & 9 & 484 & 51 & 596 & 13 \\
\hline Säulenbasalt & 1888 & 1097 & 170 & 30 & 592 & 378 & 364 & 84 \\
\hline Basaltlava & $\approx 803$ & 20 & $\because 212$ & 2 & $* 56$ & $* 58$ & $* 554$ & $* 5$ \\
\hline
\end{tabular}

Parallel zu den Beobachtungen auf den Kurzzeitserien besiedelte Balanus balanoides bevorzugt den Muschelkalk. Die Uberlebensrate war auf diesem Substrat sowie auf Granit am Ende des Versuchsjahres 1972 am höchsten (Tab. 4). Die Bevorzugung des Muschelkalks als Siedlungssubstrat ließ sich auch daran erkennen, daß die bodenreifen Cypris-Larven zuerst dieses Gestein aufsuchten. War eine Dichte von mehr als 10 Individuen pro $\mathrm{cm}^{2}$ erreicht, stieg die Zuwachsrate am Ende der Siedlungsperiode auf den bis dahin schwächer besetzten Substraten an, zum Beispiel dem ebenen, feinkörnigen Solnhofener Plattenkalk und Säulenbasalt (Abb. 5). Im Jahr 1972, einem Jahr mit hohem Siedlungsdruck bei B. balanoider, war diese Erscheinung besonders auffällig. Schwache Wind- und Wellenbewegung Anfang Mai begünstigten ebenfalls das Ansetzen der Cypris-Larven auf den letztgenannten Gesteinen.

Die Siedlungsmuster von Balanus crenatus und $B$. improvisus - beide Arten waren vorzugsweise auf den fein- bis mittelkörnigen Gesteinen Solnhofener Plattenkalk, Buntsandstein und Säulenbasalt zu finden - könnten als Folge einer Raumkonkurrenz durch $B$. balanoides gesehen werden, doch zeigen die Ergebnisse der Kurzzeitserien (Tab. 2), daß beide Arten, unabhängig von einer Konkurrenz durch $B$. balanoides, die genannten Gesteine vielfach dichter besiedelten als grobkörnige Substrate. 


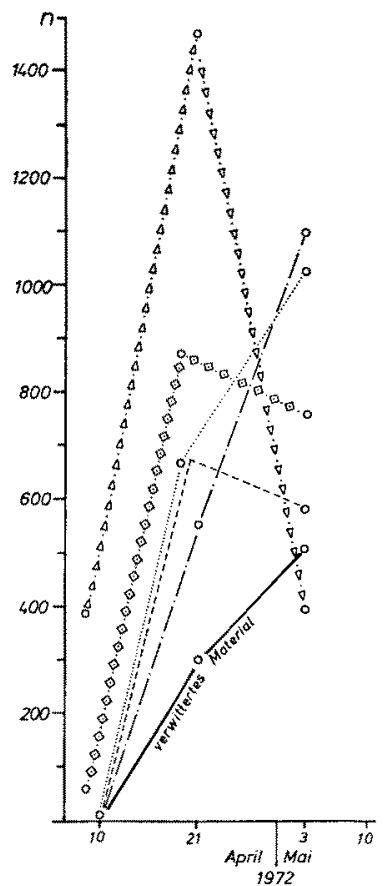

\section{Balanus balanoides}

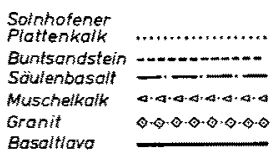

Easalliavo

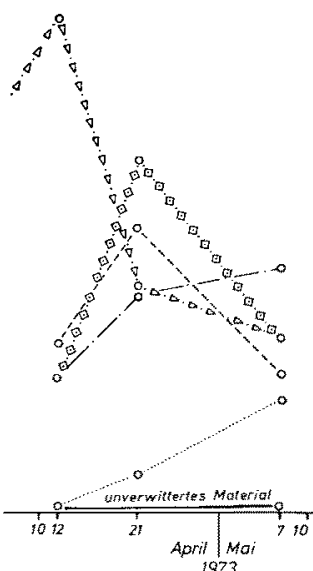

Abb. 5: Balanus balanoides. Zugang von Junggut auf den Natursteinsubstraten im Bereich der Mitteltiden-Niedrigwasserlinie. Langzeitserien der Versuchsjahre 1972 und 1973

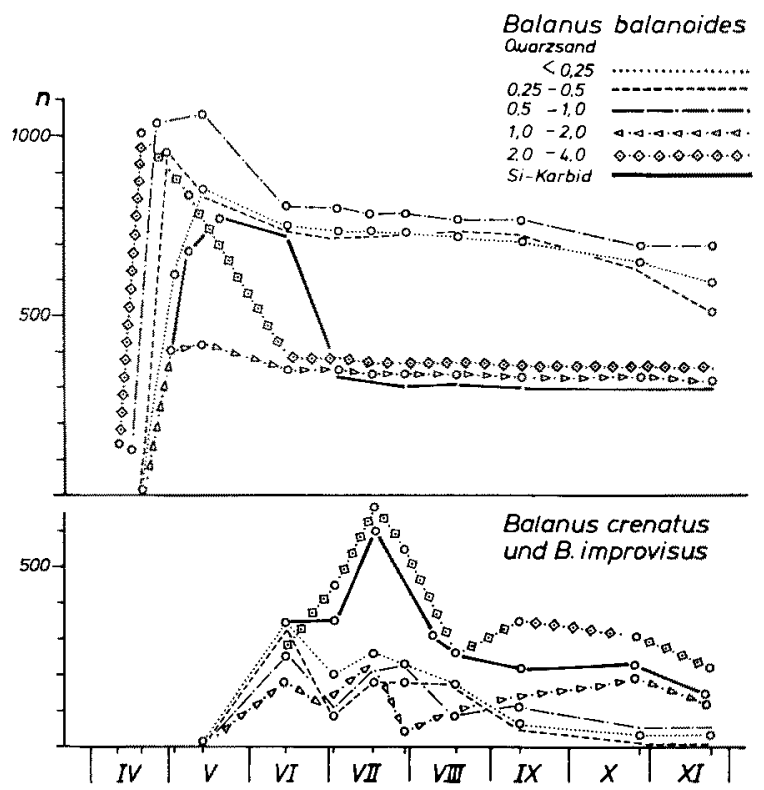

Abb. 6: Besiedlungsdynamik der Balaniden auf den Quarzsandsubstraten im Bereich der Mitteltiden-Niedrigwasserlinie, Langzeitserie des Versuchsjahres 1972 
Am Ende des Jahres 1972 (Tab. 4) war die Abundanz der drei Balanidenarten auf unregelmäßigen, rauhen Substraten (Muschelkalk, Buntsandstein und Granit) am höchsten, nämlich 5 Individuen pro $\mathrm{cm}^{2}$. Sie betrug auf der relativ glatten Oberfläche von Säulenbasalt 3,7 Individuen pro $\mathrm{cm}^{2}$ und auf dem abrasionsempfindlichen Solnhofener Plattenkalk nur 1,3 Individuen pro $\mathrm{cm}^{2}$. Auf dem unverwitterten $\mathrm{Ma}-$ terial der Basaltlava setzten sich die Balaniden erst $a b$ Juli an und erreichten eine maximale Dichte von 2,4 Individuen pro $\mathrm{cm}^{2}$. Es ist anzunehmen, daß die Oberfläche unverwitterter, naturrauher Basaltlava erst der abschleifenden Wirkung des Meerwassers unterliegen muß, um für eine Balanidenbesiedlung geeignet zu sein.

Bei den Quarzsandsubstraten, die in der Regel weniger dicht von Balaniden besiedelt wurden, ließen sich ähnliche Tendenzen wie bei den Naturgesteinen erkennen. Balanus balanoides war vorzugsweise auf feinem Grobsand und Feinkies zu finden (Abb. 6 oben), die wesentlich kleineren Cypris-Larven von B. crenatus und $B$. improvisus dagegen auf der glatten Kittmasse in den Lücken zwischen den Feinkieskörnern (Abb. 6 unten). Auf Feinsand schien ein dichter Enteromorpha-Rasen das Ansetzen der später siedelnden Balanidenarten zu behindern (vgl. Abb. 9 in Luther, 1976b). Die Uberlebenschancen von B. balanoides erwiesen sich auf feinem Grobsand am günstigsten, auch in den kleineren Fraktionsbereichen besser als auf Sandsubstraten mit mehr als $1 \mathrm{~mm}$ Korndurchmesser. Die Sterblichkeit der später siedelnden Balanidenarten war auf letzteren geringer.

Die Verteilung des Muscheljunggutes erschien auf den Natursteinsubstraten der Langzeitserie, auf der sich durch eine frühzeitige Balanidenbesiedlung nur kurzfädige Enteromorpha-Rasen entwickeln konnten, ziemlich gleichmäßig. In Ermanglung fädiger Strukturen sammelten sich die jungen Mytili kranzartig um die Opercularplatten der Balaniden an und saßen am zahlreichsten auf Individuen vom Turmhabitus, die sich in besonders dichten Balanidensiedlungen, zum Beispiel auf Granit, herausgebildet hatten. Bei Versuchsende waren die sessil gewordenen Jungtiere (Größe bis $4 \mathrm{~mm}$ ) in die Klüfte zwischen die Balanidenkegel abgewandert. Auf den Quarzsandsubstraten hatte sich Enteromorpba sp. im Feinsandbereich zu langfädigen Thalli entwickelt. Hier befand sich auch die Masse des Muscheljunggutes, während die älteren Tiere später die Lücken zwischen den Körnern des Grobsand- und Feinkiesbereichs aufsuchten oder sich zwischen Balanidenkegeln ansetzten.

Polydora ciliata siedelte auf der Langzeitserie 1972 Anfang August. Die Tiere der ersten Population vermehrten sich ständig, so daß bis Ende November die größte Dichte erreicht war (Abb. 7). $\mathrm{Zu}$ diesem Zeitpunkt herrschte noch rege Tentakeltätigkeit. Polydora ciliata bohrt in Kalkstein und in Kalkschalen lebender und toter Organismen (Hempel, 1957). Da die Mauerplatten der Balaniden ein leicht anzubohrendes Material darstellen, befanden sich die Polychaeten am zahlreichsten auf Substraten mit dichter Balanidenbesiedlung. Sie bauten dort ihre Wohnröhren so gedrängt um die Seepocken, daß deren Opercularplatten wie in einem Krater versunken schienen.

Die ungestörte Entwicklung von Polydora ciliata hing nicht nur von der Dichte der Balanidensiedlungen und der Größe ihrer Einzeltiere, sondern auch von ihrer Beständigkeit ab. Bei Muschelkalk mit besonders beständigem Balanidenbewuchs und Basaltlava, in deren Poren die Würmer ihre Wohnröhren anlegten, stieg die Abundanz 
stetig an (Abb. 7). Bei Gestein, auf dessen Oberfläche ein Bewuchs weniger gut haftete, wurde die Abundanzzunahme je nach Größe der abradierten Balanidenplacken mehr oder weniger gestört, doch glich sich der Verlust durch die hohe Vermehrungsrate des Polychaeten rasch aus. Einzig auf dem abrasionsgefährdeten Solnhofener Plattenkalk konnten sich nur schwache Siedlungen von Balaniden und Polychaeten entwickeln.

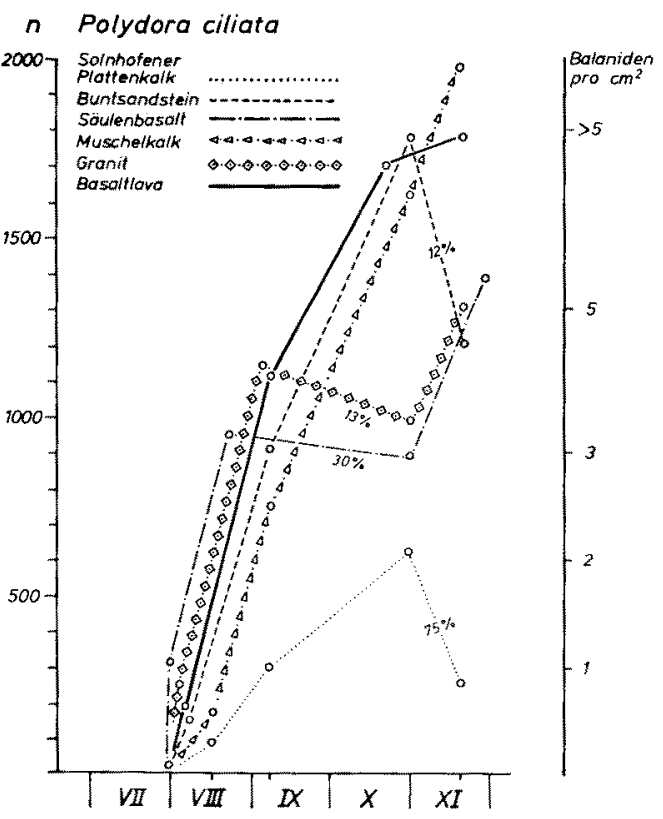

Abb. 7: Polydora ciliata. Besicdlungsdynamik auf den Natursteinsubstraten im Bereich der Mitteltiden-Nicdrigwasserlinic, Lang\%citserie des Versuchsjahres 1972. Bewuchsverluste sind in Flächenprozenten der Versuchsfelder angegeben

Durch die Befunde auf den Quarzsandsubstraten wurde deutlich, daß Polydora ciliata nicht nur bevorzugt zwischen dichtsitzenden Balanidenkegeln siedelte, sondern auch die Wohnröhren in schluchtartigen Lücken zwischen groben Grobsandkörnern und Kristallen des Siliziumkarbids anlegte. Auf dem abrasionsanfälligen Feinsandsubstrat wurde der Polychaet so rasch reduziert, daß der Verlust durch den Nachwuchs nicht ausgeglichen werden konnte.

Thecate Hydrozoen, in erster Linie Laomedea longissima, entwidkelten sich 1972 ab Mitte Juni auf allen Substraten und erreichten Ende Juli auf Buntsandstein und Basaltlava einen Bedeckungsgrad von $70 \%$, auf den restlichen Substraten nur 25 bis $40 \%$. Mitte August gingen die Kolonien zurück, dafür breiteten sich junge Kolonien von L. flexuosa auf den durch Abrasion bewuchsfrei gewordenen Flächen von Solnhofener Plattenkalk und Säulenbasalt aus. Ende November waren thecate Hydrozoen kaum noch zu finden, während athecate Kolonien der Familie Corynidae auf Buntsandstein und Basaltlava einen Bededkungsgrad von ca. 30\% erreichten. Die 
Hydrozoenkolonien zeigten auf den Quarzsandsubstraten in ihrer Entwicklung starke Schwankungen. Trotz dieser Schwankungen ließ sich erkennen, daß die Korngrößen-bereiche von 1,0 bis $4,0 \mathrm{~mm}$ bevorzugt besiedelt wurden.

Zusammenfassend lassen sich folgende Ergebnisse herausstellen: $\mathrm{Da}$ im Bereich der Mitteltiden-Niedrigwasserlinie die Abrasion den Bewuchs stärker als in den beiden anderen Gezeitenzonen beeinflußte, siedelten die untersuchten tierischen Organismen vorwiegend auf Substraten mit unregelmäßigen, rauhen Oberflächen, deren Schutz gegen Wellenbewegung eine bessere Haftung gewährleisteten. Balanus balanoides, die in diesem Bereich dominierende Art, setzte sich bevorzugt auf dem schartigen Muschelkalk und dem feinen Grobsand an, B. crenatus und B. improvisus dagegen auch auf feinkörnigen, glatten Oberflächen (Solnhofener Plattenkalk, Buntsandstein und Säulenbasalt und der glatten Kittmasse zwischen den Partikeln grober Quarzsandsubstrate). Das Verhalten der beiden letztgenannten Balanidenarten schien nicht allein durch die Konkurrenz des früher siedelnden B. balanoides bestimmt zu sein. Basaltlava erwies sich zuerst als besiedlungsfeindlich. Nachdem jedoch im Laufe des Jahres durch Abrasion die scharfkantigen Porenränder abgeschliffen waren, bildeten sich kleine Balanidengruppen.

Die Jungtiere von Mytilus edulis traten, unabhängig von der Art des Substrates, dort am zahlreichsten auf, wo sich fädige Strukturen (zum Beispiel Enteromorpha sp.) und die Orificiumränder älterer Balaniden als erhöhter Siedlungsplatz anboten. Die zum Bodenleben übergehenden Jungmuscheln suchten dagegen die Klüfte zwischen den Balanidenkegeln oder den Körnern im Grobsand- und Feinkiesbereich auf.

Polydora ciliata entwickelte sich selten als makroskopischer Primärbesiedler auf einem der Substrate, und dann meist in den Poren der Basaltlava oder in den Lücken zwischen Grobsand- und Feinkieskörnern. Der Polychaet erschien hauptsächlich als Sekundärbesiedler in älteren Balanidensiedlungen und bildete aus seinen Wohnröhren dort dichte Polster. Die Größe der Polydora-Siedlungen nahm bis November ständig zu. Sie verhielt sich proportional zur Größe der Balanidensiedlungen.

Die Kolonien thecater und athecater Hydrozoen entwickelten sich vorwiegend auf rauhen Substraten (Buntsandstein, Basaltlava, seltener Muschelkalk und Granit, daneben auf Mittelsand bis zum Korngrößenbereich von Feinkies). Diese Substrate eigneten sich für eine sichere Verankerung der Stolonen. Tubularia larynx trat nur als Irrgast auf.

\section{Tierische Besiedlung auf dem Unterwasserniveau}

Die Temperaturen waren auf dem Unterwasserniveau ausgeglichener als in den höher gelegenen Zonen. Die Lichtintensität nahm ab, ebenso die der Wellenbewegung, die an der Wasseroberfläche stärker wirkte als im Sublitoral. Es konnten sich Sinkstoffe auf den Substraten ablagern. Bewuchsverluste durch Abrasion waren geringer als im Bereich der Mitteltiden-Niedrigwasserlinie, da dort zusätzlich klimatische Einflüsse mitspielten. Nur die Oberfläche des wenig widerstandsfähigen Solnhofener Plattenkalkes blätterte stellenweise ab. Der Bewuchs auf den glattgesägten Substraten und dem feinkörnigen Quarzsand wurde am Jahresende durch Welleneinwirkung zerstört. 
Im Bewuchsbild herrschten Tiersiedlungen, vor allem solche von Balaniden, vor. Neben ihnen waren die gleichen sessilen Formen wie im Bereich der MitteltidenNiedrigwasserlinie zu finden, außerdem die Anthozoe Metridium senile, vereinzelt Kolonien der Bryozoe Electra pilosa und ein Tunicat, Molgula oculata. Das Abundanz-Verhältnis verschob sich jedoch zwischen einigen Arten; zum Beispiel dominierte Balanus crenatus vor $B$. balanoides; Lanice conchilega und Tubularia larynx, die sich auf dem Niedrigwasserniveau vereinzelt entwickelt hatten, bildeten dichte Gruppen und Kolonien. Zusammen mit den Hydrozoen traten vagile Formen als Weidegänger auf wie die Nacktschnecken Tergipes despectus, Dendronotus frondosus, Cratena autuntia, Facellina drummondi, der Amphipode Caprella linearis und der Pantopode Phoxichilidium femoratum. Sie konnten das Wachstum der Kolonien stark beeinträchtigen. Carcinus maenas und Asterias rubens waren als Aasfresser und Räuber auf allen Substraten vorhanden. Im allgemeinen setzte, Balaniden ausgenommen, die Besiedlung auf dem Unterwasserniveau wesentlich früher ein als die der gleichen Arten im Bereich der Mitteltiden-Niedrigwasserlinie. Die einzelnen Substrate unterschicden sich in Art und Dichte der Besiedlung nicht mehr so deutlich voneinander als auf dem höheren Niveau, doch ließen sich noch Differenzen feststellen.

Kurzzeitserien. Balanus balanoides besiedelte überwiegend die unregelmäßBigen Gesteine Muschelkalk, Granit und, dichter als auf dem höheren Niveau, den ebenen, jedoch durch die Korngröße oberflächenrauhen Buntsandstein. B. crenatus bevorzugte die gleichmäßigen fein- bis mittelkömigen Gesteine Solnhofener Plattenkalk, Buntsandstein und Säulenbasalt. B. improvisuts zeigte ahnliche Tendenzen wio B. crenatus (Tab. 5). Basaltlava war, mit Ausnahme des durch Verwitterung veränderten Materials, besiedlungsfeindlich.

Auf den Quarzsandsubstraten siedelten die drei Balanidenarten durchweg im Bereich des Feinsands bis zu dem des feinen Grobsands (Abb. 8). In Feinkicsbereich traten sie dagegen weit weniger auf als in gleichen Bereich auf dem Nicdrigwasserniveau.

Tabelle 5

Balanus balanoides, B. crenatus und $B$. improvisus. Zugang von Junggut auf dem Unterwasserniveau (Kurzzeitserien) in den Jahren 1971 bis 1973. Individuenzahl des Junggutes pro Versuchssubstrat

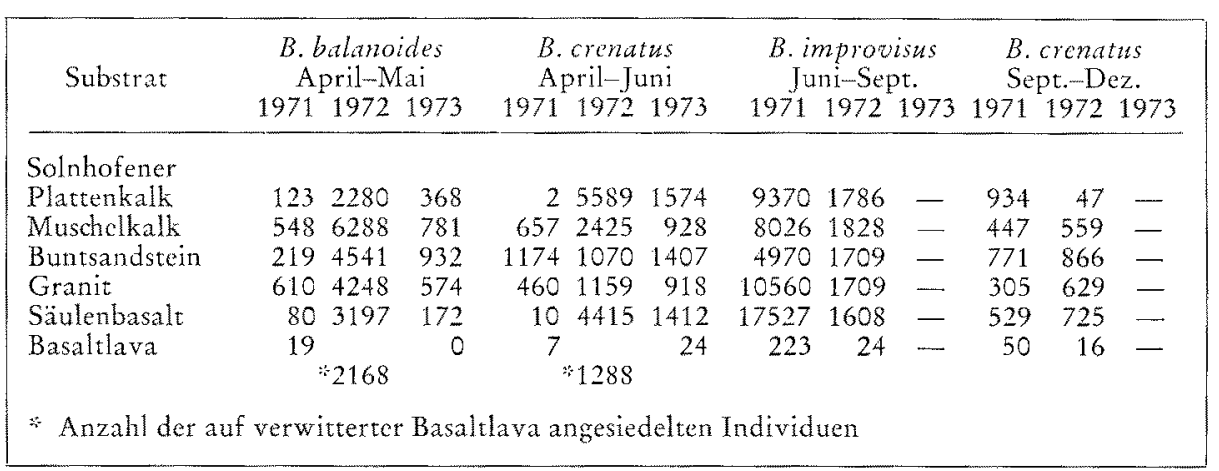


Die Anzahl von Mytilus edulis war auf dem Unterwasserniveau, verglichen mit der Abundanz des Junggutes im Bereich der Mitteltiden-Niedrigwasserlinie, verschwindend gering. Es fehlten hier die fädigen Thalli der Grünalgen, die sich im oberen Tidenbereich zu dichten Rasen entwickelt hatten, und die Balanidenkegel waren zu klein, um den jungen Muscheln als erhöhte Anheftungspunkte zu dienen.

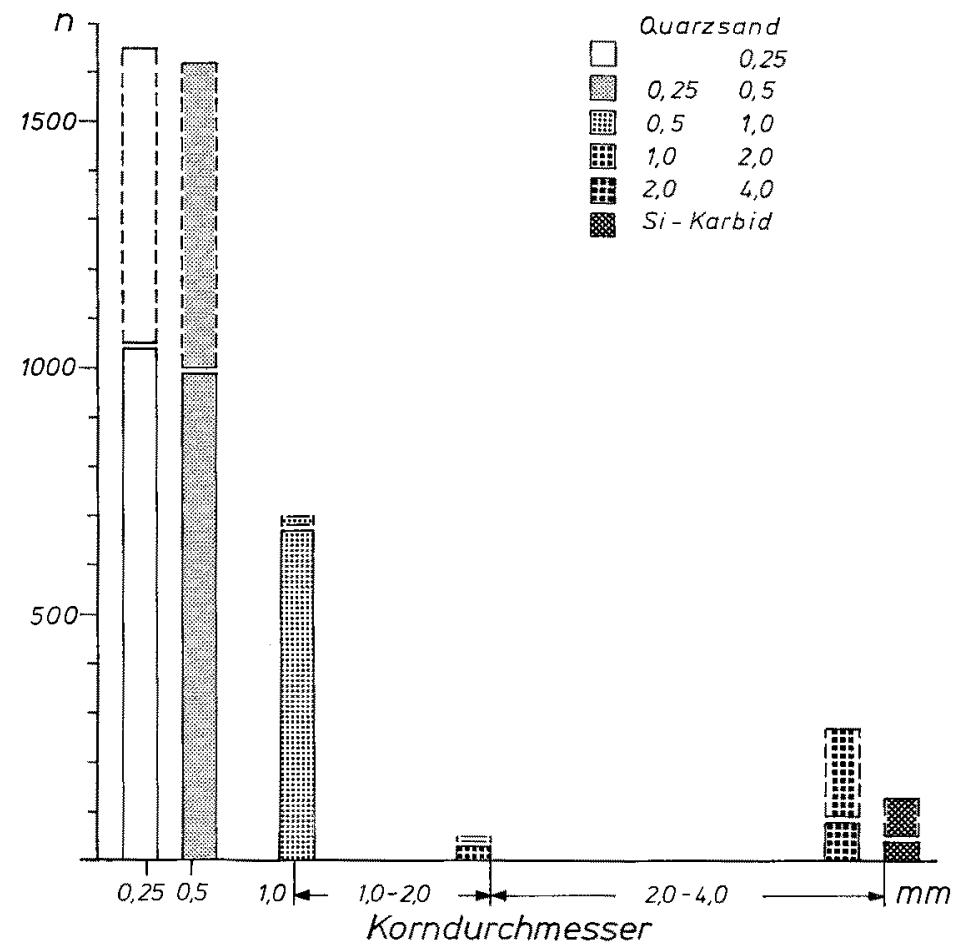

Abb. 8: Balanus balanoides, B. crenatus und B. improvisus. Zugang von Junggut auf den Quarzsandsubstraten abgestufter Korngrößen von April bis Juli 1972, Kurzzeitserien auf dem Unterwasserniveau. Kolumnen ausgezogen: Anteil von Balanus balanoides; Kolumnen gestrichelt: Anteil von Balanus crenatus und B. improvisus

Dieser Befund galt für die Substrate beider Reihen, Natursteine sowie Quarzsande. Polychaeten und Hydrozoen traten im Gegensatz zum Niedrigwasserniveau häufig als makroskopische Primärbesiedler auf. Polydora ciliata baute ihre Wohnröhren im weichen Solnhofener Plattenkalk und Muschelkalk oder in den Poren der Basaltlava. Auf den Quarzsandsubstraten fehlte sie. Hydrozoenkolonien entwickelten sich überwiegend auf unregelmäßigen und grobkörnigen Substraten, wo die Stolonen besser verankert werden konnten.

Langzeitserien. Die Befunde der Langzeitserien gleichen, speziell bei Balaniden, denen der Kurzzeitserien. Balanus balanoides dominierte auf den unregelmäßigen, grobkörnigen Gesteinen Muschelkalk und Granit, B. crenatus und B. improvisus auf den ebenen, feinkörnigen Gesteinen Solnhofener Plattenkalk und Säu- 
Tabelle 6

Balanus balanoides, B. crenatus und B. improvisus. Abundanz auf dem Unterwasserniveau (Langzeitserien) in \% pro Vensuchsfläche, bezogen auf eine maximale Dichte von 2500 Individuen

\begin{tabular}{|c|c|c|c|c|c|c|c|}
\hline Species & $\begin{array}{c}\text { Soln- } \\
\text { hofener } \\
\text { Platten- } \\
\text { kalk }\end{array}$ & $\begin{array}{c}\text { Muschel- } \\
\text { kalk }\end{array}$ & $\begin{array}{l}\text { Buntsand- } \\
\text { stein }\end{array}$ & Granit & $\begin{array}{l}\text { Säulen- } \\
\text { basalt }\end{array}$ & $\begin{array}{c}\text { Basalt- } \\
\text { lava }\end{array}$ & Zeitspanne \\
\hline \multirow{4}{*}{$\begin{array}{l}\text { Balanus } \\
\text { balanoides } \\
\text { B. crenatus und } \\
\text { B. improvisus } \\
\text { Balanus } \\
\text { balanoides } \\
\text { Balanus } \\
\text { crenatus }\end{array}$} & 44 & 100 & 60 & 73 & 43 & 4 & \multirow{4}{*}{$\begin{array}{c}\text { April bis } \\
\text { November } \\
1972 \\
\text { April bis } \\
\text { Juni } 1973\end{array}$} \\
\hline & 97 & 57 & 72 & 68 & 77 & $35 \%$ & \\
\hline & 25 & 60 & 29 & 40 & 31 & 7 & \\
\hline & 81 & 20 & 22 & 7 & 35 & 0,1 & \\
\hline
\end{tabular}

lenbasalt. Der mittelkörnige Buntsandstein wurde von den drei Arten annähernd gleich gut besiedelt (Tab. 6).

Die Unterschiede in der "Wahl" des Substrates traten jedoch im Jahre 1972, bedingt durch starken Siedlungsdruck, weniger deutlich hervor. Die Überlebensrate

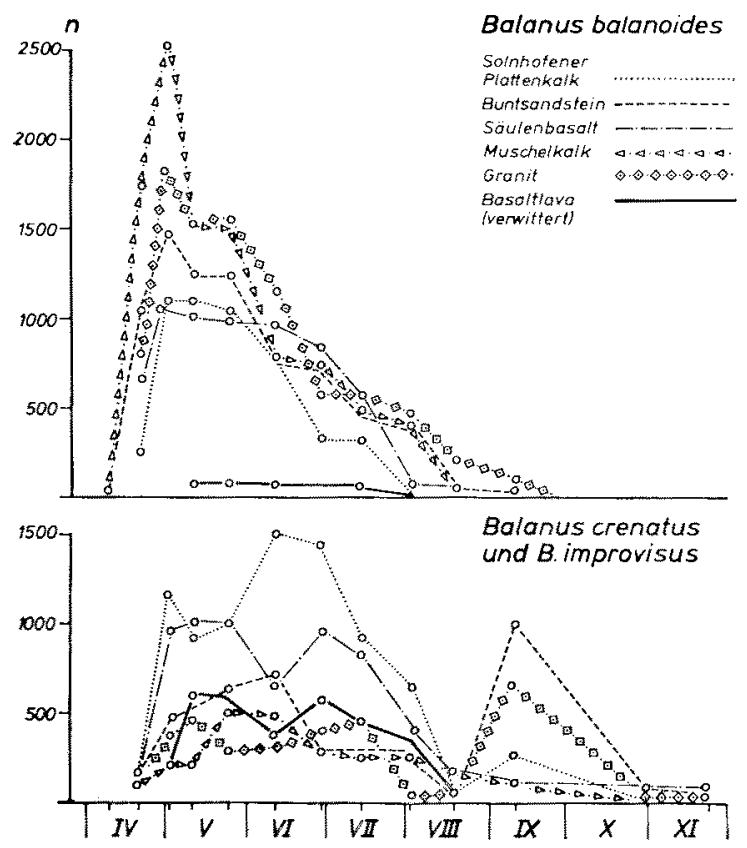

Abb. 9: Besiedlungsdynamik der Balaniden auf den Natursteinsubstraten im Versuchsjahr 1972, Langzeitserie auf dem Unterwasserniveau 
ließ sich am Jahresende nicht mehr feststellen, da Jungtiere von Asterias rubens bereits Ende August die Frïhjahrsbesiedlung vernichtet hatten (Abb. 9 und 10). Lediglich von den im Herbst neu angesiedelten Balaniden war im November noch eine geringe Anzahl vorhanden.

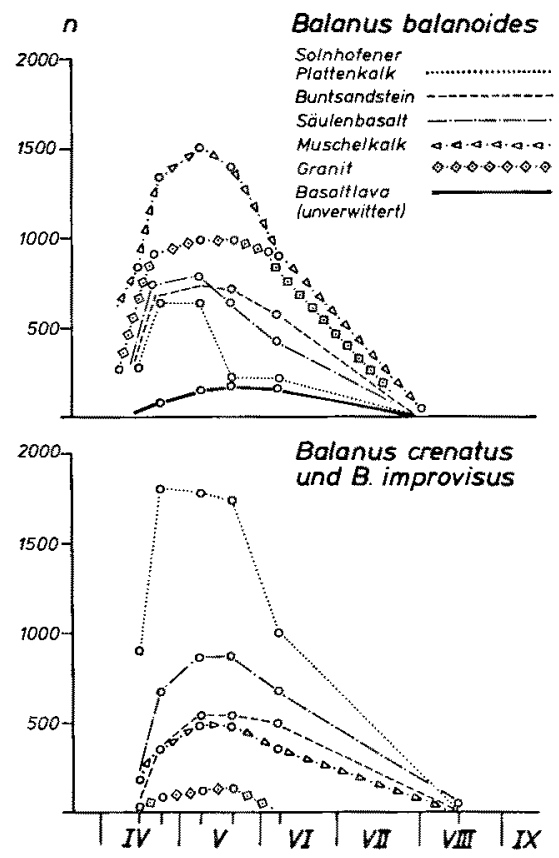

Abb. 10: Besiedlungsdynamik der Balaniden auf den Natursteinsubstraten im Versuchsjahr 1973, Langzeitserie auf dem Unterwasserniveau

Die Befunde auf den Quarzsandsubstraten entsprachen ebenfalls den bisher gemachten Beobachtungen. Balanus balanoides dominierte auf Mittel- und Grobsand, fehlte dagegen auf Feinkies. B. crenatus und B. improvisus dominierten auf Feinsand und waren zum kleineren Prozentsatz auch auf den übrigen Quarzsandsubstraten zu finden. Im Gegensatz zu Balanus balanoides setzten sie sich in den Lücken

\section{Tabelle 7}

Balanus balanoides, B. crenatus und B. improvisus. Abundanz auf dem Unterwasserniveau (Langzeitserie 1972) in \% pro Versuchsfläche, bezogen auf eine maximale Dichte von 2500 Individuen

\begin{tabular}{|lccccccc|}
\hline Species & $<0,25$ & $0,25-0,5$ & $0,5-1,0$ & $1,0-2,0$ & $2,0-4,0$ & $\begin{array}{c}\text { Silizium- } \\
\text { karbid }\end{array}$ & $\begin{array}{c}\text { Zeit- } \\
\text { spanne }\end{array}$ \\
\hline $\begin{array}{l}\text { Balanus } \\
\text { balanoides }\end{array}$ & 10 & 31 & 25 & 0 & 1 & 0 & April bis \\
$\begin{array}{l}\text { B. crenatus und } \\
\text { B. improvisus }\end{array}$ & 72 & 43 & 28 & 9 & 23 & 27 & November \\
\hline
\end{tabular}


zwischen den Feinkieskörnern an (Tab, 7), starben jedoch dort bald ab. Daher ließ sich eine nennenswerte Uberlebensrate nur auf Fein- und Mittelsand feststellen.

Im Jahr 1971 war neben der Gesteinsserie mit naturrauhen Substraten zur Kontrolle eine gleiche mit glattgesägten Oberflächen eingesetzt worden. Im Gegensatz zur naturrauhen Serie besiedelten die Balaniden, unabhängig von der Art des Gesteins, die glatten Oberflächen relativ gleichmäßig. Wenn jedoch die Besiedlung durch Abrasion zerstört wurde, was, häufiger als auf den naturrauhen Substraten, in $\mathrm{Ab}-$ ständen von ca. 4 Wochen geschah, folgte stets eine Wiederbesiedlung (Abb. 11).

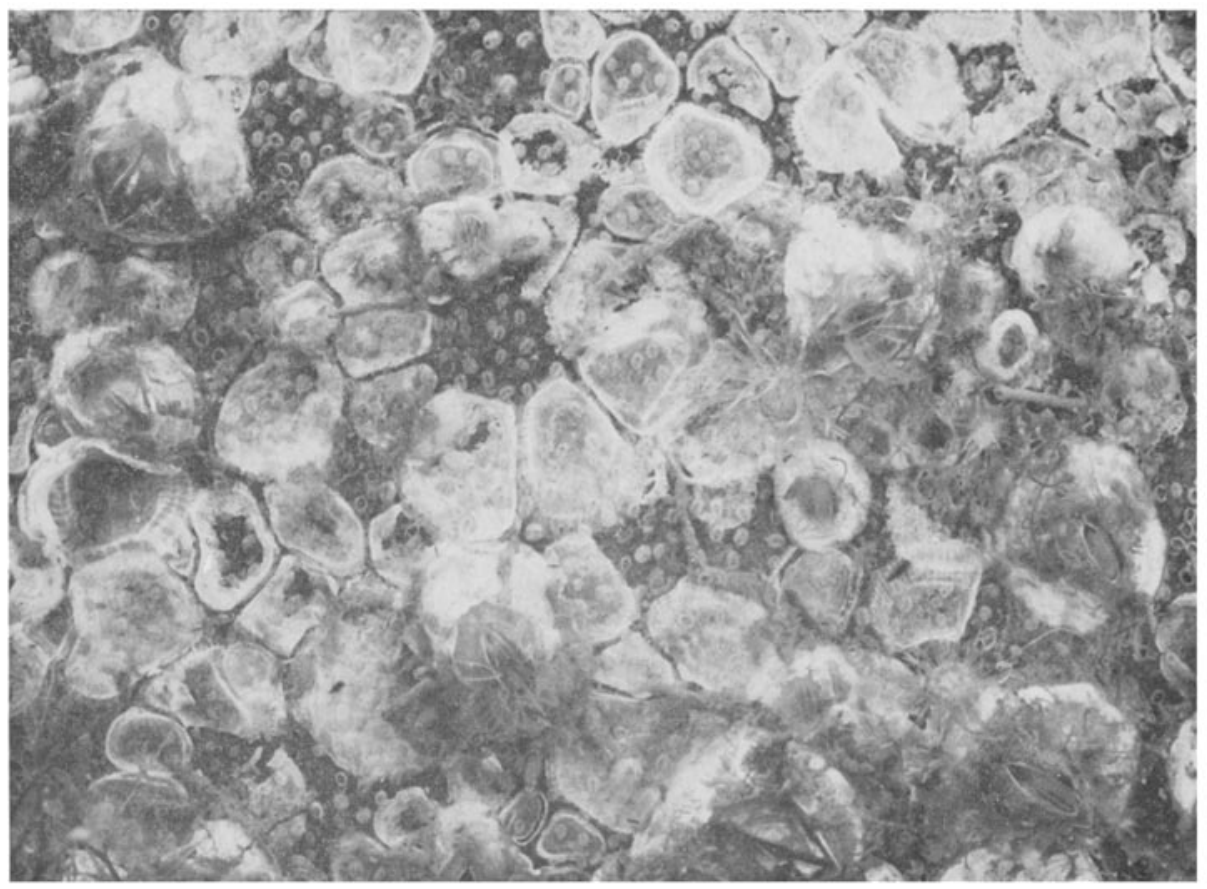

Abb. 11: Besiedlung von Balanus crenatus auf Säulenbasalt mit glattgesägter Oberfläche, Langzeitserie auf dem Unterwasserniveau im Versuchsjahr 1971

Dadurch schwankte die Abundanz ständig und crreichte erst am Jahresende auf jedem Substrat einen ungefähr gleichen Betrag von 1 Individuum pro $\mathrm{cm}^{2}$.

Das Junggut von Mytilus edulis war wie bei allen Serien auf EnteromorphaFäden und an den Orificiumrändern der Balaniden zu finden. Als die Jungmuscheln zum Bodenleben übergingen, wurden sie von Asterias rubens vernichtet, so daß bei Versuchsende keine älteren sessilen Individuen mehr anzutreffen waren.

Polydora ciliata siedelte ab Ende Mai, 3 Monate früher als auf dem Niedrigwasserniveau. Sie creichte während ihres Besiedlungsmaximums (Anfang September) eine Dichte von 500 Individuen auf den Versuchsfeld des Muschelkalks. Ende November waren nur nod wenige Individuen, meist Jungtiere, zwischen den abgestorbenen Balanidensiedlungen vorhanden, während gleichzeitig auf dem Niedrig- 
wasserniveau zwischen intakten Balanidensiedlungen ca. 2000 Würmer ausgezählt werden konnten. Den gleichen Befund ergab die Auswertung der Quarzsandsubstrate, wo Polydora ciliata ausschließlich zusammen mit dichten Balanidengruppen in den Korngrößenbereichen 0,25 bis $1,0 \mathrm{~mm}$ auftrat. Ein anderes Bewuchsbild zeigte 1971 die Serie mit den glattgesägten Natursteinen. Durch die hier besonders dichten Balanidenkrusten hatte sich der Polychaet Anfang August bis zu einem Maximum von ca. 5000 Individuen vermehrt und war im September nach der teilweisen Abrasion der Balaniden auf ein Minimum von durchschnittlich 250 Individuen zurückgegangen. Mit der Verjüngung der Balanidensiedlungen erreichte er bis November wieder einen Mittelwert von 540 Individuen. Auf der Parallelserie mit naturrauhen Gesteinen befanden sich dagegen zwischen den abgestorbenen Balanidensiedlungen nur leere Wohnröhren.

Lanice conchilega war im Juli/August 1972 mit 32 Exemplaren auf Buntsandstein vorhanden. Ihre Röhren überspannten das Versuchsfeld in ganzer Länge (bis $15 \mathrm{~cm}$ ) und richteten sich an besonders starken Tubularia-Stöckchen rechtwinklig auf. Da sich auf Säulenbasalt ebenfalls große Tubularia-Kolonien entwickelt hatten, siedelte der Polychaet auch hier zahlreich. Einen ähnlichen Befund ergab die Untersuchung der Feinsand- und Feinkiessubstrate. Auf den glattgesägten Gesteinsoberflächen, die 1971 eingesetzt waren, fehlten Polychaet und Hydrozoe. Außer der Befestigungsmöglichkeit schien der Materialfang zum Röhrenbau eine wesentliche Rolle zu spielen. Dieser konnte durch Absanden des Substrats oder Trübeablagerungen zwischen den Unregelmäßigkeiten der Oberfläche erleichtert werden.

Laomedea longissima überzog ab Juni die unregelmäßigen Gesteine Muschelkalk und Basaltlava vollkommen mit ihren Kolonien. Als im August Laomedea flexnosa auftrat, vergrößerten sich die von Hydrozoen bewachsenen Flächen auch auf feinkörnigen Substraten, besonders auf Solnhofener Plattenkalk. Da sich thecate Hydrozoen zeitiger entwickelt hatten als athecate, waren letztere auf Gesteinen mit weniger ausgedehnten Laomedea-Kolonien $\mathrm{zu}$ finden. Die gleiche Beziehung bestand beim Hydrozoenbewuchs der Quarzsandserie. Die zuerst siedelnden Laomedea-Arten dehnten sich auf den grobkörnigen Substraten aus. Sie wurden in dem Maße, in dem sie sich verringerten, durch die athecaten Formen Tubularia larynx und Coryne sp. ersetzt. Auf den gesägten Gesteinen entwickelte sich Laomedea sp. erst dann gleichmäßig gut, wenn die Oberflächen durch Einwirkung des Meerwassers aufgerauht waren, während Tubularia larynx fehlte.

Zusammenfassend lassen sich folgende charakteristische Befunde für die Besiedlung auf dem Unterwasserniveau aufzeigen. Durch die Abnahme der Lichtintensität verminderte sich der Algenbewuchs zugunsten tierischer Besiedlungen. Da gleichzeitig die Oberflächenturbulenz des Wassers abnahm, traten die Bewuchsunterschiede zwischen den einzelnen Substraten weniger deutlich hervor als auf dem Niedrigwasserniveau. Die Entwicklung der Organismen wurde, vermehrt durch Siedlungsdruck, stärker von Konkurrenten als von abiotischen Faktoren bestimmt.

Balanus balanoides bevorzugte zum Siedeln unregelmäßige Gesteine und war auch häufig auf mittelkörnigen Substraten zu finden. $B$. crenatus und B. improvisus setzten sich überwiegend auf fein- und mittelkörnigen Substraten an. Da Asterias rubens den Balanidenbewuchs unterhalb der Mitteltiden-Niedrigwasserlinie bis August 
vernichtet hatte, konnte bei Versuchsende nur die Uberlebensrate der Herbstbesiedlung festgestellt werden. Die Jungtiere von Mytilus edulis suchten für die kurze Dauer ihrer hemisessilen Phase fädige Algenthalli und die Orificiumränder größerer Balaniden auf. Als sie zum Bodenleben übergingen, wurden sie von Asterias rubens vernichtet. Polydora ciliata siedelte 3 Monate früher als auf dem Niedrigwasserniveau. Bei Versuchsende befanden sich, wenige Jungtiere ausgenommen, nur leere Wohnröhren auf den Substraten, während gleichzeitig die Besiedlung auf dem Niedrigwasserniveau ihren Höhepunkt erreichte. Lanice conchilega trat am zahlreichsten zusammen mit Tubularia-Stöckchen auf. Hydrozoen vermochten jede relativ rauhe Oberfläche am besten zu besiedeln. Die Abundanz der einzelnen Arten hing. bedingt durch den Zeitpunkt der Besiedlung, von der Raumkonkurrenz untereinander $a b$.

\section{DISKUSSION UND SCHLUSSFOLGERUNGEN}

Aus den Befunden lassen sich folgende allgemeine Schlüsse ableiten. Ein Hartsubstrat kann diejenigen siedelnden Organismen direkt beeinflussen, die auch - abgesehen von dem in dieser Arbeit nicht berïcksichtigten Primärschleim - direkt mit der Oberfläche in Berührung kommen wie Balaniden, bodenreife junge Miesmuscheln, bohrende Polychaeten und Hydrozoen. Die epibiotischen Sekundärbesiedler dagegen, die auf Art und Dichte des makroskopischen Primärbewudses angewiesen sind, unterliegen auf diesem Umweg einem indirckten Einfluß des Substrats (hemisessiles Junggut der Miesmuscheln, der Polychaet Polydora ciliatta, einige Weidegänger und Räuber).

\section{Direkter Einfluß des Substrates auf Balanidenbewuchs}

Der direkte Einfluß eines Substrats basiert auf mehreren Faktoren wie Struktur, Härtegrad, Farbe, Widerstandsfähigkeit gegen Abrasion und Erosion. Diese Faktoren prägen das Siedlungsmuster auf dem Hochwasserniveau besonders stark. Sie verlieren mit zunehmender Wassertiefe an Wirkung. Am deutlichsten ließ sich bei den Untersuchungen der Einfluß der Oberflächenstruktur verfolgen. Hierbei ist zu unterscheiden: Ein Mikrorelief, gekennzeichnet durch die Korngröße des Gesteins, und ein Makrorelief, gemessen an größeren Höhenunterschieden pro $\mathrm{cm}^{2}$. Andere Strukturelemente wie Dichte und Porosität des Hartsubstrats bestimmten bei den Untersuchungen die Art des tierischen Siedlungsmusters weniger deutlich als zum Beispiel die der untersuchten Algen (Luther, 1976b).

Die Balaniden Balanus balanoides, B. crenatus und B. improvisus, beherrschten als dominierende Arten der Makrofauna das Bewuchsbild. Über ihr allgemeines Siedlungsverhalten ist folgendes bekannt: Sie bevorzugen rauhe Oberflächen (Pyefinch, 1948; Barnes \& Powell, 1953); die Abundanz ist auf unebenen Stellen größer als auf glatten (Küh1, 1951); besonders glatte Flächen werden gemieden (Hatton, 1938; Knight-Jones, 1953); nach Experimenten von Pomerat \& Weiss (1946) besetzten sie 
faserige Texturen am dichtesten; Algenbewuchs (Moore, 1934; Crisp, 1958) und Muddansammlungen (Knight-Jones, 1953) beeinträchtigen die Entwicklung der Balaniden, während Substrate, auf denen sich lebende Cirripedier oder ihre Plattenreste befinden, zuerst besiedelt werden (Knight-Jones, 1955; Crisp \& Meadows, 1962, 1963; Crisp, 1964).

An speziellem Besiedlungsverhalten wurde beschrieben: Balanus balanoides meidet Substrat von geringem Härtegrad, während er ein solches in geschützten Lagen, wo die Wasserturbulenz geringer ist, gelegentlich besiedelt (Crisp \& Southward, 1958). Nach Moore \& Kitching (1939) ist er auf Kalk der oberen Kreide nicht zu finden, besetzt dagegen die darin enthaltenen Flintknollen und den harten Portlandkalk. Wohlenberg (1937) berichtet von Siedlungen dieses Cirripediers auf Miesmuschelschalen. Er fehlt jedoch bei Bewuchs von Fucus mytili. Nach Schäfer (1952) meidet Balanus balanoides bis auf Ausnahmefälle organischen Kalk, insbesondere den der eignen Species. $B$. crenatus ist weniger wählerisch in der Art des Substrats. Er reagiert positiv auf jeden organischen Kalk und setzt sich so dicht auf den Mauerplatten der eignen Species an, daß ein sogenanntes Knäuelwachstum entsteht (Schäfer, 1952). $B$. improvisus zeigt ein ähnliches Siedlungsverhalten. Er besiedelt außerdem besonders glatte Oberflächen von Leder, Kunststoffen und Plexiglas (Kühl, 1951, 1958).

Das Besiedlungsverhalten der Balaniden auf den Substraten der eigenen Versuchsreihen ließen folgende grundsätzliche Tendenzen erkennen: Im Bereich starker Wasserturbulenz (Hochwasser- und Niedrigwasserniveau) setzten sich die bodenreifen Cypris-Larven überwiegend auf unregelmäßigen Oberflächen an (Muschelkalk, Granit und Buntsandstein), wo sie Schutz fanden. Mit zunehmender Wassertiefe vergrößerte sich die Abundanz auf ebenflächigen Gesteinen (Solnhofener Plattenkalk, Säulenbasalt). Außer diesem allgemeinen Verhalten zeichneten sich Differenzen bei den einzelnen Species ab. So überwog Balanus balanoides durchweg auf unregelmäßigen, mittel- bis grobkörnigen Substraten (Muschelkalk, Granit und Buntsandstein), die sublitoralen Species, B. crenatus und B. improvisus, dagegen häufig auf glatten, feinkörnigen (Solnhofener Plattenkalk, Säulenbasalt). Vielleicht wird die "Substratwahl" durch die Ausbildung der Basalplatten begünstigt. Balanus balanoides besitzt eine membranöse, die sublitoralen Species besitzen dagegen verkalkte Basalplatten. Es wäre zu untersuchen, wie weit letztere den Mauerring besser als Balanus balanoides auf einem glatten Substrat verankern können. Die Differenzierung zeigte sich deutlicher auf den Befunden der Kurzzeitserien. Auf den Langzeitserien wurde sie oft durch Konkurrenz im Laufe der Sukzession verwischt oder wenn bei starkem Siedlungsdruck die Cypris-Larven auf ein benachbartes, weniger günstiges Substrat übergriffen. Letztere Beobachtung wurde bereits von Knight-Jones \& Stevenson (1950) und Knight-Jones (1953) beschrieben. Bei den eigenen Versuchen waren die Cypris-Larven bei starkem Siedlungsdruck sogar auf den Thalli von Porphyra purpurea zu finden. Die Jungtiere brachen bei einem basalen Durchmesser von $3 \mathrm{~mm}$ heraus, wobei der Umriß der Basalplatte als Loch im pflanzlichen Gewebe sichtbar blieb. Ein einziges Substrat erwies sich in der Reihe der Naturgesteine als besiedlungsfeindlich, die Basaltlava. Ihre Oberfläche war von Poren (Durchmesser 0,1 bis $4,0 \mathrm{~mm}$ ) übersät, die scharfkantigen, dicht aneinanderschließenden Ränder verhinderten offensichtlich, daß sich Cypris-Larven ansetzten. Wo jedoch eine Kalkdruse oder 
nachträglich eingedrungenes Material die Oberfläche umgewandelt hatten, konnten kleine "Siedlungsinseln" entstehen, von denen aus sich die Balaniden langsam verbreiteten. Die Cypris-Larven drückten sich an die äußeren adulten Tiere an und vergrößerten somit den Umfang der Siedlung.

Zur Kontrolle wurde auf dem Unterwasserniveau neben der Serie mit naturrauhen Gesteinen eine gleiche mit glattgesägten Oberflächen eingesetzt. Es zeigte sich auf den verschiedenen glattgesägten Substraten, selbst auf Basaltlava, kaum ein Unterschied in der Abundanz der Balaniden. Diese Beobachtung führte zu der Oberlegung, daß die Oberflächenstruktur besonders stark eine Besiedlung beeinflussen kann. Um die Wirkung der Oberflächenrauhigkeit zu untersuchen, wurde eine Serie mit Quarzsanden abgestufter Korngrößen hergestellt. Diese Serie, deren Substraten ein Makrorelief fehlte, ließ wiederum ein spezifisches Siedlungsverhalten bei den $\mathrm{Ba}$ laniden erkennen. Balantw balanoides dominierte im Korngrößenbereich 0,25 bis $1.0 \mathrm{~mm}$ - entsprechende Korngrößen sind in der Mehrzahl bei Muschelkalk, Granit und Buntsandstein zu finden -. Die sublitoralen Balanidenarten waren im Korngrößenbereich $<0,25$ bis $0,5 \mathrm{~mm}$ am häufigsten. Diese Fraktionen ïberwiegen bei Solnhofener Plattenkalk, Buntsandstein und Säulenbasalt. Die besonders kleinen CyprisLarven von $B$. crenatts und $B$. improvisus siedelten auch zahlreich auf dem glatten Epoxydharz, das die Räume zwischen den Feinkieskörnern (Durchmesser 2,0 bis $4,0 \mathrm{~mm}$ ) ausfüllte. Sie starben $a b$, wenn das enge Lumen zwischen den Köornern ein weiteres Wachstum verhinderte. Die Korngröße wirkte in diesem Fall nicht als Gradmesser der Oberflächenrauhigkeit, sondern als Merkmal für die Reliefhöhe. Die $A$ bundanz war, entsprechend dem Grad der Wasserturbulenz, im Bereich der MitteltidenNiedrigwasserlinie größer als auf dem Unterwasserniveau. Im Korngrößenbereich 1,0 bis $2,0 \mathrm{~mm}$ traten dagegen wenig Balaniden auf. Weder das Lumen zwischen den Körnern noch die Flächen der Körner selbst schienen zu genügen, daß sich CyprisLarven ansetzen konnten (vgl. auch die Porenstruktur der Basaltlava).

Werden Substrateigenschaften und die entsprechenden Besiedlungsbefunde aufeinander bezogen, lassen sich die in den Abbildungen 12 bis 14 wiedergegebenen Schemata aufstellen. Auf dem Hochwasserniveau wirken sich bei den Balaniden als besonders begrenzend aus: die Temperaturerhöhung während des Trockenliegens und das Fehlen einer ausreichenden Reliefhöhe als Schutz gegen Wasserturbulenz. Auf dem Niedrigwasserniveau tritt zu den genannten begrenzenden Eigenschaften die Abrasionsanfälligkeit des Bewuchses hinzu. Letztere ist an die Struktur des Substrats gebunden (Abblättern oder Absanden von Schichten, schlechte Verankerung der Organismen auf glatten Oberflächen). Nach den Schemata (Abb. 12, 13) wird Solnhofener Plattenkalk im Hochwasser- und Niedrigwasserbereich als ebenes, abrasionsanfälliges Material nicht oder nur wenig dauerhaft besiedelt. Dieser Befund stimmt mit den Beobachtungen von Moore \& Kitching (1939) überein, nach denen Balanus balanoides auf Kreidekalk fehlt. Die Substrateigenschaften des letzteren beeinflussen offensichtlich die Balanidenbesiedlung ähnlich wie die des Solnhofener Plattenkalks. Im umgekehrten Sinn besteht bei dem beständigeren Portlandkalk und Muschelkalk die gleiche Übereinstimmung. Der Befund, daß im Unterwasserbereich die Abundanz der Balaniden auf ebenen, glatten Substraten zunimmt (Abb. 14), scheint nicht nur mit der abnehmenden Wasserturbulenz, sondern auch mit der Fähigkeit der sublitoralen Bala- 


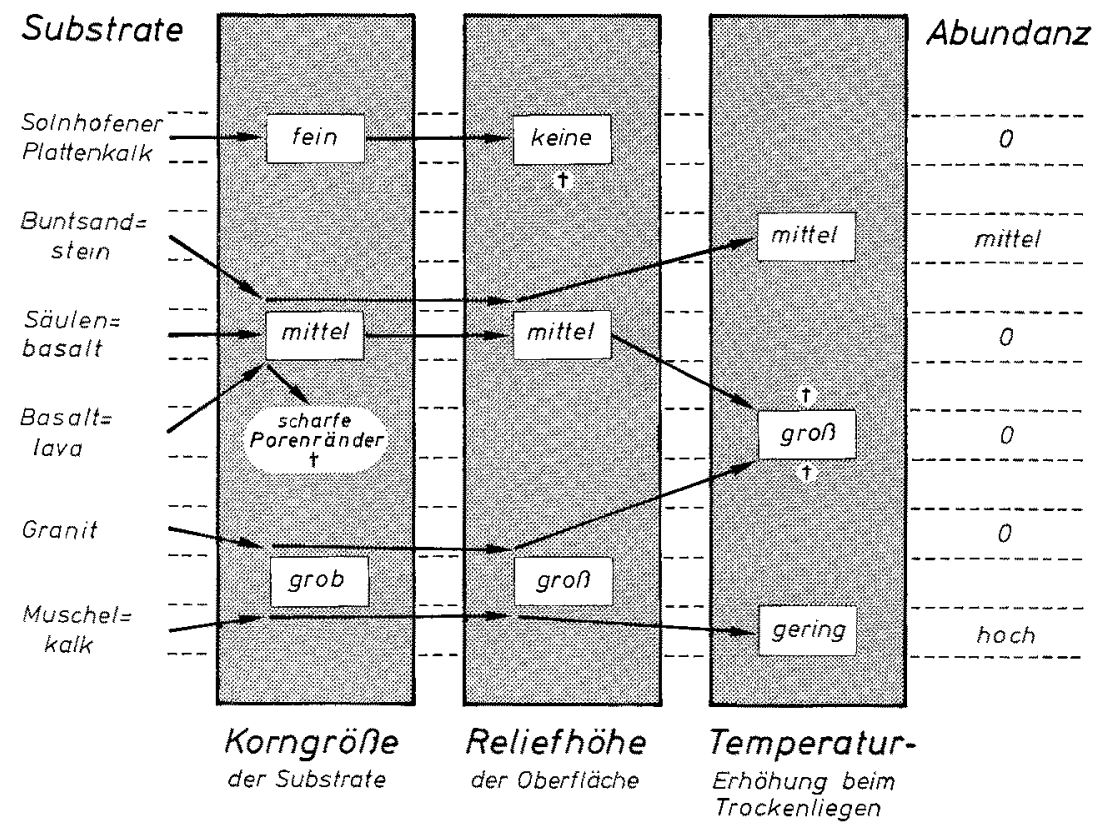

Abb. 12: Einfluß wichtiger physikalischer Eigenschaften der Gesteine auf die Besiedlung von Balanus balanoides im Bereich der Mitteltiden-Hochwasserlinie. Korngrößendurchmesser: $\leq 4 \mathrm{~mm}$; Reliefhöhe: $\leq 10 \mathrm{~mm}$ pro $\mathrm{cm}^{2}$; Temperaturerhöhung beim Trockenliegen: $\leq 12 \mathrm{C}^{0}$. Das Kreuz bedeutet Absterben der Jungtiere

nidenarten zusammenzuhängen, glatte Oberflächen gut besiedeln zu können (vgl. Kühl, 1951).

Der Einfluß vom Härtegrad und von der Farbe der untersuchten Substrate auf die Balanidenbesiedlung zeigte sich weitaus undeutlicher als der der Oberflächenstruktur. So unterschieden sich zum Beispiel Muschelkalk (Härtegrad 3) und Granit (Härtegrad 6-7) kaum in der Abundanz der siedelnden Balaniden. Auch der Einfluß der Farbe blieb gering, abgesehen vom Hochwasserbereich, wo sich dunkles Gestein bei Sonnenbestrahlung intensiver erwärmte als helles. Die Farbe als optisch stimulierender Faktor wurde auf dem Niedrigwasser- und Unterwasserniveau bei Granit sichtbar, da die suchenden Cypris-Larven die dunklen Gemengteile zahlreicher aufsuchten als die hellen. Diese Beobachtung ist eine weitere Bestätigung für die seit langem bekannte phototaktische Fähigkeit der Cypris-Larven (Visscher, 1928; Weiss 1947; Se-gal, 1970; de Wolf, 1973).

Im Gegensatz zu den Balaniden hing die Besiedlung thecater Hydrozoen mehr von der Oberflächenrauhigkeit als von der Reliefhöhe des Substrats ab. So bedeuteten zum Beispiel scharfkantige Strukturen kein Hindernis beim Siedeln. Den fehlenden Schutz gegen Wasserturbulenz ersetzten die Kolonien durch die Widerstandsfähigkeit ihrer feingliedrigen, biegsamen Stämmchen. Zudem ist die Wasserturbulenz unterhalb der Mitteltiden-Niedrigwasserlinie, wo sich die Hydrozoe überwiegend entwickelt, abgeschwächt. Die lang dahinkriechenden Stolonen fanden genügend Halt, wenn die 


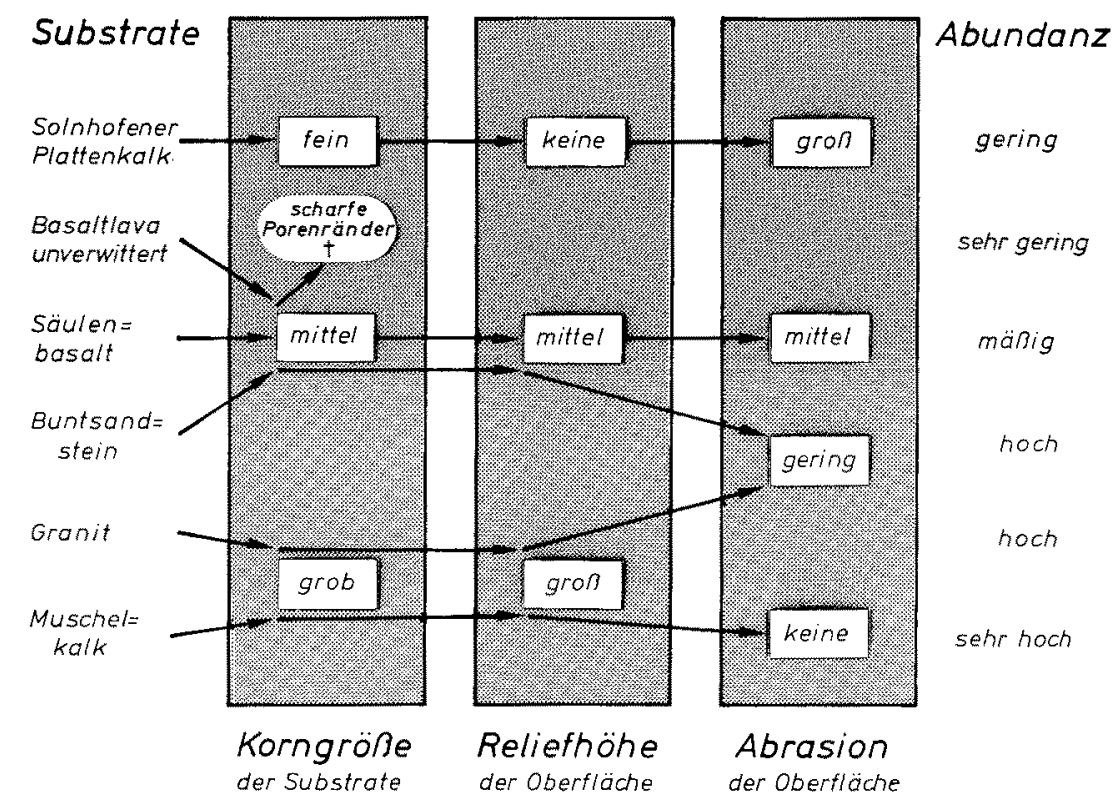

Abb. 13: Einfluk widtiger physikalischer Eigenschaften der Gesteine auf die Besiedlung von Balanus balanoides im Bereid der Mitteltiden-Niedrigwasserlinie. Korngrökendurchnesser: $\leq 4 \mathrm{~mm}$; Reliefhöhe: $\leq 10 \mathrm{~mm}$ pro $\mathrm{cm}^{2}$; Abrasion der Oberfläche: $\leq 90$ Flächenprozente des Versuchsfeldes. Das Kreuz bedeutet Absterben der Jungtiero

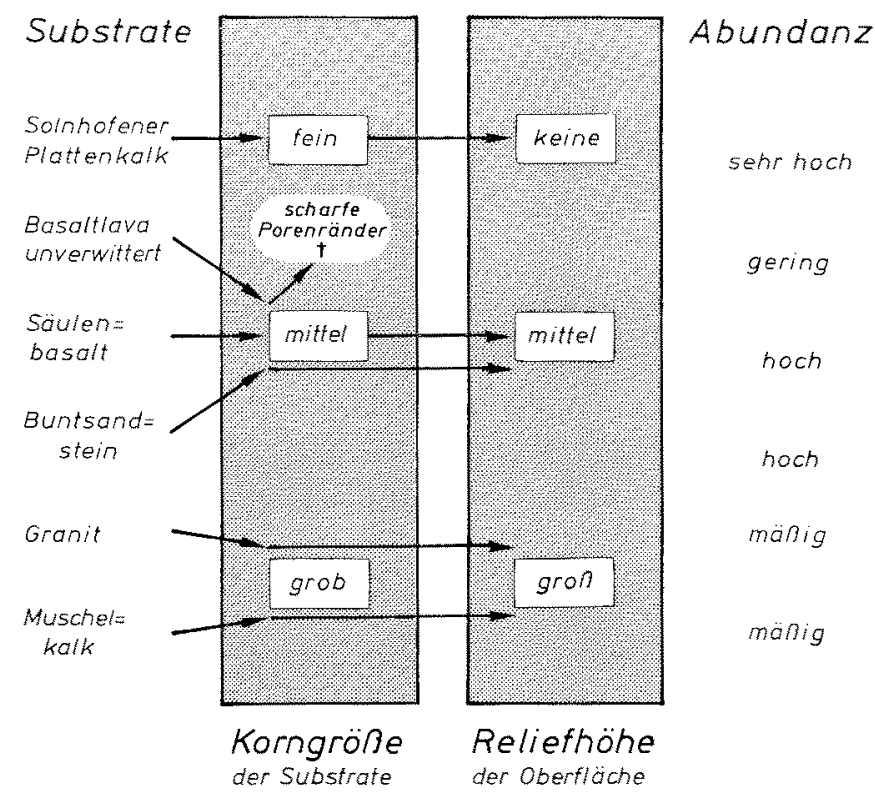

Abb. 14: Einflu13 wichtiger physikalischer Eigenschaften der Gesteine auf die Besiedlung von Balanus crenatus und $B$. improvisus im Unterwasserbereich. Korngrößendurchmesser: $\leq 4 \mathrm{~mm}$; Reliefhöhe: $\leq 10 \mathrm{~mm}$ pro $\mathrm{cm}^{2}$. Das Kreuz bedeutet Absterben der Jungtiere 
Oberfläche durch die Art der Korngröße rauh genug war. Daher gediehen die Kolonien am schnellsten auf rauhen Gesteinen wie Muschelkalk, Buntsandstein und Basaltlava und daher war die Abundanz auf den Quarzsandsubstraten der Korngröße proportional. In der Zeit hohen Siedlungsdrucks, zum Beispiel während des Entwicklungsmaximums von Laomedea flexuosa, wurden auch die feinkörnigen, glatten Gesteine Solnhofener Plattenkalk und Säulenbasalt dicht von den zarten Stolonen dieser Hydrozoe überzogen. Die gleiche Beobachtung traf für die Entwicklung der athecaten Tubularia larynx auf den Substraten der Kurzzeitserien zu. Auf den Substraten der Langzeitserien wurde diese Entwicklung durch die Konkurrenz der frïher siedelnden thecaten Hydrozoen in der Hinsicht beeinflußt, daß Tubularia larynx auf weniger rauhe Oberflächen $(<0,25 \mathrm{~mm}$ Korngröße) ausweichen mußte.

\section{Einfluß makroskopischen Primärbewuchses auf Sekundärbesiedler}

Einige Formen der Makrofauna zeigten sich als Sekundärbesiedler deutlich von Art und Dichte eines makroskopischen Primärbewuchses abhängig wie zum Beispiel Jungmuscheln von Mytilus edulis und der bohrende Polychät Polydora ciliata.

Es ist bekannt, daß junge Miesmuscheln während einer kurzen hemisessilen Phase, bevor sie zum Bodenleben übergehen, fädige Algen und bäumchenförmige Hydrozoenkolonien (Verwey, 1951; Bayne, 1964) oder die Mauerkronen großer Balanidenkegel (Caspers, 1949; Kühl, 1951) als erhöhte Siedlungsplätze aufsuchen. Diese Beobachtungen ließen sich während beider Versuchsjahre (1971 und 1972) in der Weise bestätigen, daß die Jungtiere in großer Zahl auf den Substraten siedelten, die dicht mit den oben genannten Formen bewachsen waren. Daher traten die jungen Mytili im Bereich der Mitteltiden-Niedrigwasserlinie überwiegend in den Enteromor$p h a-$ Rasen von Solnhofener Plattenkalk und Buntsandstein oder den buckelförmigen Balanidenkrusten von Muschelkalk und Granit auf. Sie verteilten sich dagegen gleichmäßig über alle Substrate im Unterwasser-Bereich, die einen entsprechend gleichmäßigen Bewuchs von Hydrozoen und adulten Balaniden aufwiesen. Die Beziehung zwischen Substrat, Primärbewuchs und Jungmuscheln wurde durch die Befunde der Kurzzeitserien besonders deutlich. Hier konnten sich nur schnellwüchsige Enteromorpha-Arten auf Solnhofener Plattenkalk und Buntsandstein (vgl. Luther, 1976b) ausbilden. Das Entwicklungsmaximum der Alge mußte jedoch mit der hemisessilen Phase von Mytilus edulis zusammentreffen (Ende Juni bis Anfang Juli). Diese Obereinstimmung bestand $1971 \mathrm{im}$ Bereich der Mitteltiden-Niedrigwasserlinie, fehlte hingegen auf dem Unterwasserniveau. Daher betrug die Abundanz der Jungmuscheln auf letzterem nur den 10. Teil. Im Jahr 1972 kehrte sich das Verhältnis um, nachdem sich die Grünalge auf dem Unterwasserniveau üppiger als im Bereich der Mitteltiden-Niedrigwasserlinie entwickelt hatte.

Der Polychaet Polydora ciliata bohrt in weichem, kalkigem Material anorganischer oder organischer Herkunft (Hempel, 1957; Krumbein \& van der Pers, 1974), oder heftet sich an Gegenständen an, die aufrecht im Lockersediment stecken (Schäfer, 
1962). Bei den eigenen Versuchen war Polydora ciliata kaum als Primärbesiedler auf den vertikal verlaufenden Substratoberflächen vorhanden. Die Jungwürmer suchten dann in dem durch Wasserturbulenz gekennzeichneten Tidenbereich die Poren der Basaltlava auf, oder bauten ihre Wohnröhren entlang der Schichtkanten weicher Sedimentgesteine (Solnhofener Plattenkalk, Muschelkalk), wo sie auch ihre Bohrspuren hinterließen. Dagegen entwickelten sie sich auf Substraten mit dichtem Seepockenbestand zu rasch anwachsenden Siedlungen. Nach Daro (1969) ist Polydora ciliata an exponierten, vertikalen Wänden nur im Schutz eines makroskopischen Primärbewuchses wie zum Beispiel Miesmuscheln zu finden. Auf den vertikal angebrachten Substraten der eigenen Versuchsreihen boten die Balanidensiedlungen einen annähend gleichwertigen Schutz wie Miesmuscheln. Die Abundanz des Polychaeten war der Abundanz der Balaniden proportional. Den gleichen Effekt hatte Hempel (1959) beobachtet.

Mehrere Faktoren schienen die "Wahl" des tierischen Substrats zu bestimmen: der bercits erwähnte Schutz gegen Wasserturbulenz, das leicht anzubohrende Material der Balanidenkegel, die Sedimentablagerungen dazwischen, die nach Daro \& Polk (1973) das Ansetzen stimulieren, und nicht zuletzt die Erscheinung, die Riedl (1971) mit Xenorheophilie bezeichnet. Zur Xenorheophilie neigen solche Organismen, die sich im Bereich des Atem- und Nahrungswasserstroms größerer Strudler und Filtricter ansetzen und davon profitieren. Auch der Wasserstrom der schlagenden Balandenzirren könnte die Jungw ürmer angeregt haben, sich rund um die Mauerkegel und innerhalb der Orificiumränder anzusiedeln. Da sich junge, hemisessile Miesmuscheln an den gleichen Stellen anspinnen, wäre auch hicr eine Form von Xenorheophilic anzunehmen.

Eine anderc Bezichung, die Xenorheophilie betreffend, ließe sich aus der Weiterentwicklung der vergesellschafteten Tierformen ableiten. Nachdem auf dem Unterwasserniveau Asterias rubens im August die Balaniden vernichtet hatte, zeigte sich in den ausgeräuberten Siedlungen außer einigen Jungtieren kein aktiver Polychaet mehr. Scine lecren Wohnröhren (Durchmesser bis $0,5 \mathrm{~mm}$ ) umgaben die ausgefressenen Mauerkronen. Der Schutz der toten Balanidenkegel genügte offensichtlich nicht, den Fortbestand der Polychaetensiedlung zu sichern. Ohne den Zirrenwasserstrom des tierischen Substrats schien Polydora ciliata an diesem Standort keine optimalen Lebensbedingungen mehr zu finden. Es ließ sich nicht feststellen, ob die letzte Brut nur abgewandert war oder ob sich das Zerstörungswerk des Scesterns direkt auf den Polychaeten ausgewirkt hatte. Auf dem Niedrigwasserniveau dagegen, wo sich die Balaniden ungestört entwickeln konnten, nahm auch die Abundanz von Polydora ciliata bis zum Jahresende zu. Die Abhängigkeit von einer intakten Primärbesiedlung wurde bereits von Schütz \& Kinne (1955) erwähnt: "Polydora ciliata kommt bevorzugt vergesellschaftet mit Mytilus edulis vor. Dort, wo diese Muschel ihr Vorkommen einstellt, tritt $P$. ciliata trotz offenbar zusagender Bedingungen nur noch selten auf." Dasselbe Zitat läßt sich für die Vergesellschaftung von Polydora ciliata mit Balanus sp. anwenden. Da Asterias rubens durch seine räuberische Tätigkeit in Laufe des Sommers die untere Verbreitungsgrenze der Balaniden bestimmte, legte er somit auch die des vergesellschafteten Polychaeten fest.

Die Befunde beider Versuchsjahre bestätigen dic Beobachtungen Paines (1974), nach denen biotische Einflüsse stärker als abiotische dic Struktur der Lebensgemein- 
schaften im Bereich ständiger Wasserbedeckung verändern. In höher gelegenen Tidenzonen überwiegt dagegen der Einfluß physikalischer Umweltfaktoren.

\section{ZITIERTE LITERATUR}

Barnes, H. \& Powell, H. T., 1953. The growth of Balanus balanoides (L.) and B. crenatus Brug. under varying conditions of submersion. J. mar. biol. Ass. U. K. 32, 107-127.

Bayne, B. L., 1964. Primary and secondary settlement in Mytilus edulis L. J. Anim. Ecol. 33, (5) $3-523$.

Caspers, H., 1949. Die Bewuchsgemeinschaft an der Landungsbrücke der Nordseeinsel Spiekeroog und das Formproblem von Balanus. Zool. Jb. (Systematik) 78, 237-322.

Crisp, D. J., 1958. Distribution of intertidal organisms. J. mar. biol. Ass. U. K. 37, 157-208.

- 1964. Surface chemistry, a factor in the settlement of marine invertebrate larvae. Bot. Gothoburg. 3, 51-65.

- \& Meadows, P. S., 1962. The chemical basis of gregariousness in cirripedes. Proc. R. Soc. (B) 156, 500-520.

- 1963. Adsorbes layers; the stimulus to settlement in barnacles. Proc. R. Soc. (B) 158, 364-387.

- \& Southward, A. J., 1958. Distribution of intertidal organisms, J. mar. biol. Ass. U. K. 37, $157-208$.

Daro, M. H., 1969. Etude écologique d'un brise-lames de la côte belge. I. Description et zonation des organismes. Annls Soc. r. zool. Belg. 99, 111-152.

- \& Polk, P., 1973. The autecology of Polydora ciliata along the Belgian coast. Neth. J. Sea Res. 6, $130-140$.

Hatton, H., 1938. Essais de bionomie explicative sur quelque espèces intercodiales d'algues et d'animeaux. Annls Inst. océanogr. Monaco 17, 241-248.

Hempel, C., 1957. Zur Okologie einiger Spinoiden (Polychaeta sedentaria) der deutschen Küsten. Kieler Meeresforsch. 13, 275-288.

- 1959. Uber das Festsetzen der Larven und die Bohrtätigkeit der Jugendstadien von Polydora ciliata (Polychaeta sedentaria). Helgoländer wiss. Meeresunters. 7, 82-92.

Knight-Jones, E. W., 1953. Laboratory experiments on gregariousness during setting in Balanus balanoides and other barnacles. J. exp. Biol. 30, 584-598.

- 1955. The gregarious setting of the barnacles as a measure of systematic affinity. Nature, Lond. 174, 1-266.

- \& Stevenson, J. P., 1950. Gregariousness during settlement in the barnacle Elminius modestus (Darwin). J. mar. biol. Ass. U. K. 29, 281-297.

Krumbein, W. E. \& van der Pers, J. N. C., 1974. Diving investigations on biodeterioration by sea-urchins in the rodky sublittoral of Helgoland. Helgoländer wiss. Meeresunters. 26, 1-17.

Kühl, H., 1951. Vergleichende biologische Untersuchungen über den Hafenbewuchs. Zool. Anz. (Suppl.) 15, 233-244.

Luther, G., 1976a. Bewuchsuntersuchungen auf Natursteinsubstraten im Gezeitenbereich des Nordsylter Wattenmeeres. Helgoländer wiss. Meeresunters. 28, 145-166.

- 1976b. Bewuchsuntersuchungen auf Natursteinsubstraten im Gezeitenbereich des Nordsylter Wattenmeeres: Algen. Helgoländer wiss. Meeresunters. 28, 318-351.

Moore, H. B., 1934. The biology of Balanus, I. J. mar. biol. Ass. U. K. 19, 851-868.

- \& Kitching, J. A., 1939. 'The biology of Chthamalus stellatus (Poli). J. mar. biol. Ass. U. K. $23,521-541$.

Paine, R. T., 1974. Intertidal community structure. Experimental studies on the relationship between a dominant competitor and its principal predator. Oecologia 15, 93-120.

Pomerat, C. M. \& Weiss, C. M., 1946. The influence of texture and composition of surface on the attachment of sedentary marine organisms. Biol. Bull. mar. biol. Lab., Woods Hole 91, 57-65. 
Pyefinch, K. A., 1948. Notes on the biology of cirripedes. J. mar. biol. Ass. U. K. 27, 464-503. Riedl, R., 1971. Water movement, general aspects of water movement, animals. In: Marine ecology. Ed. by O. Kinne. Wiley-Interscience, London, 1 (2), 1123-1156.

Schäfer, W., 1952. Biologische Bedeutung der Ortswahl bei Balaniden-Larven. Senckenbergiana $33,235-246$.

- 1962. Aktuo-Paläontologie nach Studien in der Nordsee. Kramer, Frankfurt a. M., 666 pp.

Schütz, L. \& Kinne, O., 1955. Uber die Mikro- und Makrofauna der Holzpfähle des NordOstseekanals und der Kieler Förde. Kieler Meeresforsch. 11, 110-135.

Segal, E., 1970. Light, animals. In: Marine ecology. Ed. by O. Kinne. Wiley-Interscience, London, $1(1), 159-211$.

Verwey, J., 1951. Het Ontstaan van Mosselbanken. Naturkunde, Koniglijke Nederl. Akad. van Wetenschappen 8, DI, LX.

Visscher, J. P., 1928. Reaction of the cyprid-larvae of barnacles at the time of attachment. Biol. Bull. mar. biol. Lab., Woods Hole 54, 327-336.

Weiss, C. M., 1947. The effect of illumination and stage on tide on the attachment of barnacles cyprids. Biol. Bull, mar. biol. Lab., Woods Hole 93, 240-249.

Wohlenberg, E., 1937. Die Wattenmeer-Lebensgemeinschaften im Königshafen von Sylt. Helgoländer wiss. Meeresunters. 1, 1-92.

Wolf, P. dc, 1973. Ecological obscrvations on the mechanisms of dispersal of barnacle larvae during planctonic live and settling. Neth. J. Sea Res, 6, 1-129.

Anschrift der Autorin: Dr. Gertrand Luther

Biologische Anstalt Helgoland

(Litoralstation)

D-2282 List/Sylt

Bundesrepublik Deutschland 